\title{
The Luminosity Profiles of Brightest Cluster Galaxies
}

\author{
C.J. Donzelli, H. Muriel \\ IATE, Observatorio Astronómico OAC, Laprida 854, X5000BGR, Córdoba, Argentina \\ Consejo Nacional de Investigaciones Científicas y Técnicas (CONICET), Avenida Rivadavia 1917, \\ C1033AAJ, Buenos Aires, Argentina \\ J. P. Madrid \\ Centre for Astrophysics and Supercomputing, Swinburne University of Technology, Hawthorn, VIC 3122, \\ Australia
}

\begin{abstract}
We have derived detailed $\mathrm{R}$ band luminosity profiles and structural parameters for a total of 430 brightest cluster galaxies (BCGs), down to a limiting surface brightness of 24.5 magarcsec $^{-2}$. Light profiles were initially fitted with a Sérsic's $R^{1 / n}$ model, but we found that $205(\sim 48 \%)$ BCGs require a double component model to accurately match their light profiles. The best fit for these 205 galaxies is an inner Sérsic model, with indices $n \sim 1-7$, plus an outer exponential component.

Thus, we establish the existence of two categories of the BCGs luminosity profiles: single and double component profiles. We found that double profile BCGs are brighter $(\sim 0.2 \mathrm{mag})$ than single profile BCG. In fact, the Kolmogorov-Smirnov test applied to these subsamples indicates that they have different total magnitude distributions, with mean values $M_{R}=-23.8 \pm 0.6 \mathrm{mag}$ for single profile BCGs and $M_{R}=-24.0 \pm 0.5 \mathrm{mag}$ for double profile BCGs. We find that partial luminosities for both subsamples are indistinguishable up to $r=15 \mathrm{kpc}$, while for $r>20 \mathrm{kpc}$ the luminosities we obtain are on average $0.2 \mathrm{mag}$ brighter for double profile BCGs. This result indicates that extra-light for double profile BCGs does not come from the inner region but from the outer regions of these galaxies.

The best fit slope of the Kormendy relation for the whole sample is $a=3.13 \pm 0.04$. However, when fitted separately, single and double profile BCGs show different slopes: $a_{\text {single }}=3.29 \pm 0.06$ and $a_{\text {double }}=2.79 \pm 0.08$. Also, the logarithmic slope of the metric luminosity $\alpha$ is higher in double profile BCGs $\left(\alpha_{\text {double }}=0.65 \pm 0.12\right)$ than in single profile BCGs $\left(\alpha_{\text {single }}=0.59 \pm 0.14\right)$. The mean isophote outer ellipticity (calculated at $\mu \sim 24 \mathrm{mag} \mathrm{arcsec}^{-2}$ ) is higher in double profile BCGs $\left(e_{\text {double }}=0.30 \pm 0.10\right)$ than in single profile BCGs $\left(e_{\text {single }}=0.26 \pm 0.11\right)$. Similarly, the mean absolute value of inner minus outer ellipticity is also higher in double profile BCGs

On the other hand, we did not find differences between these two BCGs categories when we compared global cluster properties such as the BCG-projected position relative to the cluster X-ray center emission, X-ray luminosity, or BCG orientation with respect to the cluster position angle.
\end{abstract}

Subject headings: galaxies: clusters: general — galaxies: elliptical and lenticular

\section{Introduction}

Brightest cluster galaxies (BCGs) are the most massive and most luminous galaxies in the uni- verse today. BCGs are so massive $\left(M_{\text {star }}>\right.$ $\left.10^{11} M_{\odot}\right)$ that their formation and evolution is closely tied to the large scale structure of the universe (Conroy et al. 2007). Semi-analytical mod- 
els show how BCGs are formed through complex hierarchical merging of many small galaxies and originate within the densest dark matter halos of primordial density fluctuations. BCGs reside only in overdense regions of the Universe such as galaxy clusters and groups where merging occurs at a high rate over cosmic time (de Lucia \& Blaizot 2007). It is precisely the accretion of numerous stellar systems that give the BCGs their apparently homogenous properties. For instance, their total luminosities are relatively constant and can be used as standard candles (Lauer \& Postman 1992).

For several decades the luminosity profiles of elliptical galaxies were modeled with the empirical $R^{1 / 4}$ de Vaucouleurs law (de Vaucouleurs 1948). However Lugger (1984) and Schombert (1986) showed that most elliptical galaxies have a flux excess at large radii with respect to the $R^{1 / 4}$ model. Schombert (1987) modelled the BCG light profiles with a power law rather than the de Vaucouleurs law underscoring the different nature of BCGs and standard elliptical galaxies.

A model used virtually by every author in recent years to fit the light profiles of a wide range of stellar systems is the generalization of the de Vaucouleurs law introduced by Sérsic $(1963,1968)$. The Sérsic model in the form $R^{1 / n}$ is a mixture of bulge and disk components using only three free parameters $\left(\mu_{e}, r_{e}\right.$, and $\left.n_{s}\right)$ instead of four $\left(\mu_{e}, r_{e}\right.$, $\mu_{0}$, and $r_{0}$ ) (see Section 3 and the comprehensive review of Graham and Driver 2005).

More recently, several papers have suggested that a simple Sérsic $R^{1 / n}$ law does not properly model the luminosity profile of some elliptical (usually cD) galaxies. Gonzalez et al. (2003, 2005) found that the best fit to the light profiles of 30 BCGs was a double $R^{1 / 4}$ de Vaucouleurs profile. Seigar et al. (2007) studied the light profiles of five $\mathrm{cD}$ galaxies and showed that a Sérsic plus an exponential function are necessary to accurately reproduce an inner and an outer component present in their surface brightness profiles. Donzelli et al. (2007) estimated that roughly half of 82 elliptical galaxies belonging to the $3 \mathrm{CR}$ radio catalog also require a Sérsic + exponential model to properly fit their light profiles. Using numerical simulations Hopkins et al. (2009) propose that dissipational mergers are at the origin of the double components light profiles in the core of elliptical galaxies. According to their models a violent re- laxation of stars whose parent galaxies participate in the merger is responsible for the creation of an outer component while a central starburst gives rise to the inner component.

We use a homogeneous and uniquely large sample of ground-based imaging of Abell clusters to carefully examine the luminosity profiles of 430 BCGs and determine the best fitting function and structural parameters. This is key to properly constrain dynamical models and the merging history of these galaxies.

The paper is organized as follows. In Section 2, we present the observations and reductions, while in Section 3 we describe data processing. Light profile fitting procedure and structural parameters are discussed in Section 4, while in Section 5 we discuss the results and in Section 6 we summarize the conclusions.

\section{Observations}

BCG images used in this work were provided by M. Postman (STScI) who kindly gave us access to the raw data. They were obtained under photometric conditions using the KPNO $2.1 \mathrm{~m}$ and $4 \mathrm{~m}$ telescopes, and the CTIO $1.5 \mathrm{~m}$ telescope between 1989 November and 1995 April over a total of 13 observing runs (see Table 1). Five of these runs are described in more detail in Postman \& Lauer (1995). Briefly, all the images were acquired in the Kron-Cousins $R_{c}$ band and have typically exposure times of 200-600 s. During these runs seeing conditions were very good to fair, namely, FWHM $=1 "-2 "$, and nights were photometric.

In order to flat field the images a series of dome flats were obtained each night. This allowed for flatfielding with a typical accuracy better than $0.5 \%$ of the sky level. Photometric calibration was obtained by observing 10-15 Landolt (1983) standard stars per night. This also enabled us to calculate extinction coefficients and to check the zero point on each night of the observing runs. The overall photometric accuracy was better than $0.02 \mathrm{mag}$, much smaller than the typical errors of the BCG photometric parameters, which are more sensitive to background subtraction, and fitting models.

One of the key points of this homogeneous sample is that approximately 50 BCGs were observed in common between the different runs. Many of 
these BCGs were actually observed in five or more runs. This not only allowed us to verify and compare reductions for all observing runs, but also to improve the accuracy of the luminosity profiles as discussed in the next section. The rms scatter for the integrated magnitudes of the galaxies is 0.03 mag, while for the luminosity profiles at $\mu_{R}=22.5$ mag $\operatorname{arcsec}^{-2}$ is $0.11 \mathrm{mag}^{\operatorname{arcsec}^{-2}}$.

\section{Data Processing and Sky Level}

All images were processed following the standard recipes: bias and flat field corrections using IRAF routines. After this process we carefully inspect the images in order to determine the best method to subtract the sky background. Gauging the sky is a crucial step since sky subtraction has a significant influence on the faint end of the luminosity profiles and therefore on the structural parameters we derive. In most cases a twodimensional first-degree polynomial was sufficient to give an accurate fit to the sky, and we used the residuals distribution to estimate the uncertainty of the sky level $\sigma_{s k y}$. The importance of sky cleaning is summarized in detail in Coenda et al. (2005).

Similar tests were made to measure the effect of seeing on the luminosity profiles. The effects of seeing dictate that the minimum radius for a suitable fit to the luminosity profile is $r=1.5 \times \mathrm{FWHM}$, this is particularly true for large galaxies, i.e., apparent radius greater than $\sim 12 \times$ FWHM (Coenda et al. 2005). We show in detail in the next section that BCG galaxies were relatively bright and extended and in most cases the apparent radius of the galaxy was greater than $20^{\prime \prime}$.

\section{Luminosity Profiles and Profile Fitting}

We use the ELLIPSE routine (Jedrzejeswki, 1987) within Space Telescope Science Analysis System (STSDAS) to extract the luminosity profiles of the BCGs. We apply this routine to the processed, sky subtracted images. In many cases galaxy overlapping is an issue due to the crowded fields around BCGs. To solve this, we apply the technique described in Coenda et al. (2005) which consists in masking the overlapping regions before profile extraction. Then we obtain the luminosity profile and structural parameters (center co- ordinates, ellipticity, and position angle of the isophotes), and construct a model BCG galaxy that is subtracted from the original image. The residual image is then used to extract the luminosity profile of the overlapping galaxies. The process is repeated several times until the profile of the target galaxy converges.

Isophote fitting was performed down to a count level of $2 \sigma_{s k y}$; i.e., the fitting procedure was stopped when the isophote level was around twice the background dispersion (pixel-to-pixel variance), which in our case corresponds to surface magnitudes between $\mu_{R} \sim 23.5-24.5 \mathrm{mag}$ $\operatorname{arcsec}^{-2}$, depending on the observational run when the cluster was observed.

For each cluster, we usually obtain the luminosity profiles for the three brightest galaxies in the field. This preliminary selection is made by eye. In those cases where the selection is not obvious, we also obtain additional luminosity profiles, i.e., for the five brightest galaxies. The final BCG selection is made through the galaxy metric luminosity, that is the luminosity enclosed in a radius of $14.5 \mathrm{kpc}$, we also included galaxy redshift to ensure cluster membership. Redshift data were obtained from the NASA/IPAC Extragalactic Database (NED).

As discussed in the introduction, there are a wide variety of functions to perform luminosity profile fitting. We adopted the Sérsic profile $R^{\beta}$, where the concentration parameter $\beta=1 / n$ is the inverse of the Sérsic index (Sérsic 1968):

$$
I(r)=I_{e} \exp \left\{-b_{n}\left[\left(\frac{r}{r_{e}}\right)^{\beta}-1\right]\right\} .
$$

In this equation $I_{e}$ is the intensity at $r=r_{e}$ the radius that encloses half of the total luminosity (also known as the effective radius or half-light radius). In this context $b_{n}$ can be calculated using $b_{n} \sim 2 n-0.33$ (Caon et al. 1993).

We use the NFIT1D routine within STSDAS to find the coefficients that best fit the light profiles of each galaxy. This task uses a $\chi^{2}$ minimization scheme to fit the best non linear functions to the light profiles tables we obtained with ELLIPSE (Schombert \& Bothun 1987). The fitting procedure is carried out only in the portion of the galaxy surface brightness profile where the signalto-noise ratio was greater than $3(S / N>3)$. We 
did this in order to avoid the regions at the faint end of the luminosity profiles, in which errors are large. Moreover, we have also excluded the inner 3"-4" of the luminosity profiles to avoid the blurring effects of seeing (see the test described in Coenda et al. 2005).

Errors on the structural parameters were calculated following the method described by Coenda et al. (2005). Briefly, this technique consists of creating test images with model galaxies that have known luminosity profile parameters. Then we artificially add and subtract to those images a constant value corresponding to $\sigma_{s k y}$. Finally we extract and refit the new luminosity profile as explained above. These newly obtained parameters give us the respective upper and lower limits.

To a first approximation a single Sérsic model provides a good fit to the light profile of our sample of BCGs, as shown by Graham et al. (1996). However, for almost half of the sample we noticed that a single Sérsic function fails to properly reproduce the BCGs surface brightness in the range $\sim 22.0$ mag $\operatorname{arcsec}^{-2}$. Note that this case is very similar to that presented in La Barbera et al. (2008, their Figure 18). It is evident that the Sérsic function do not properly fit the luminosity profiles of these elliptical galaxies in the inner $4^{\prime \prime}$, where the residuals are almost $\sim 0.3 \mathrm{mag} \operatorname{arcsec}^{-2}$.

It is not necessary to have very deep luminosity profiles, as in the case of Seigar et al. (2007), to realize that for certain galaxies, even in regions of bright surface luminosity, a single Sérsic model cannot account for the concave shape of the luminosity profile.

For these galaxies their light profiles were best fitted adding to the Sérsic model of Equation (1) an outer exponential function (Freeman, 1970):

$$
I(r)=I_{0} \exp \left(-\frac{r}{r_{0}}\right) .
$$

In this equation $I_{0}$ is the central intensity and $r_{0}$ is the length scale. The inclusion of this equation in the fitting function does not necessarily means that the galaxy has a disk component in the usual sense of rotation-supported stellar system.

We have chosen the exponential function since it is the simplest function to account for the "extra-light" observed in the above mentioned galaxies. It is worth to mention that we also tried with a second Sérsic function since it has three degrees of freedom instead two. However, in terms of the $\chi^{2}$ coefficient it is not better than the Sérsic plus exponential fit. Figure 1 shows a good example: the case of BCG A0690 where clearly a single Sérsic function cannot account for the concavity of the luminosity profile. Even when the fitting becomes adequate at the faint end of the profile, the model cannot reproduce the surface magnitude in the interval between 7 and $15 \mathrm{kpc}$, which corresponds to a surface magnitude in the range of 21-22 $\mathrm{mag} \operatorname{arcsec}^{-2}$. Error bars in this region have approximately the size of the squares. On the other hand, Figure 2 shows a much better fit due to the inclusion of the exponential component (dashed line) in the fitting model. We show that the 21-22 mag $\operatorname{arcsec}^{-2}$ region is now in perfect agreement with the model and the fitting functions can also properly describe the faint end of the luminosity profile. In fact, the reduced $\chi^{2}$ coefficient we obtain with a Sérsic + Exponential model is about one order of magnitude smaller than those obtained with the single Sérsic fit. As a general rule, for most of those cases that are initially fitted with the single Sérsic function and in which $n>8$ and $r_{e}>300 \mathrm{kpc}$, it is necessary to include the exponential component to obtain a proper fit.

Intensity parameters are then converted into surface brightness, expressed in mag $\operatorname{arcsec}^{-2}$ by the equation $m=-2.5 \log (I)$, while units of $r_{e}$, and $r_{0}$ are converted to kpc. Errors for $r_{e}$ and $r_{0}$ are smaller than $15 \%$ while for $\mu_{e}$, and $\mu_{0}$ errors are below 0.20 mag $\operatorname{arcsec}^{-2}$. Total luminosities of both Sérsic and the exponential components are finally computed using the derived photometric parameters and integrating separately both components of Equations (1) and (2) as follows:

$$
L=\int_{0}^{\infty} I(r) 2 \pi r d r
$$

which yields

$$
L_{\text {Sersic }}=I_{e} r_{e}^{2} \pi \frac{2 \exp (b)}{\beta k^{2 / \beta}} \Gamma(2 / \beta)
$$

for the Sérsic component and

$$
L_{e x p}=2 \pi I_{0} r_{0}^{2}
$$

for the exponential component. $\Gamma(2 / \beta)$ is the gamma function. Total apparent magnitudes 
are then converted into absolute magnitudes. Throughout this paper we assume a Hubble constant $H_{0}=70 \mathrm{~km} \mathrm{~s}^{-1} \mathrm{Mpc}^{-1}$ together with $\Omega_{M}$ $=0.27$ and $\Omega_{\Lambda}=0.73$.

\section{Results and Discussion}

Table 2 lists the photometric parameters obtained through the fitting procedure described in Section 3 for all BCGs of the sample. Columns 16 list the Abell BCG name, Sérsic parameters $\mu_{e}$, $r_{e}, n$, exponential parameters $\mu_{0}$ and $r_{0}$. Columns 7-10 give absolute magnitude of the Sérsic component, absolute magnitude of the exponential component, total absolute magnitude of the BCG and Sérsic to exponential component light ratio. Column 11 contains the logarithmic slope of the metric luminosity or $\alpha$ parameter which is defined as $\alpha=d\left(\log \left(L_{m}\right)\right) / d\left(\log \left(r_{m}\right)\right)$, where $L_{m}$ is the total BCG luminosity within a circular aperture of radius $r_{m}$ centered on the BCG nucleus. Following Postman \& Lauer (1995) we have calculated this parameter at $\mathrm{r}=14.5 \mathrm{kpc}$. Columns $12-15$ list the inner ellipticity (measured at 10" ), outer ellipticity


and outer position angles of the isophotes where the ellipticities are measured. Position angles are from north to east and the typical observed errors are $\sim 5^{\circ}$, while typical errors for ellipticities are 0.06. Finally, column 16 lists the metric absolute magnitude also calculated at $\mathrm{r}=14.5 \mathrm{kpc}$.

The data above show that 225 out of 430 BCGs, or $52 \%$ of the sample, have a single Sérsic luminosity profile, while the remaining 205 BCGs (48\%) need a double component of Sérsic + exponential to properly fit their luminosity profiles. We note that we find 27 galaxies ( $\sim 6 \%$ of the whole sample) that have $n<1.5$ for the inner Sérsic component, which is nearer to an exponential rather than a de Vaucoleurs profile $(n \sim 4)$. Moreover, all but three, have double component luminosity profiles. This is particularly interesting since it has been suggested that BCGs usually have high Sérsic indices (Graham et al. 1996). However, Seigar et al. (2007) observe an inner exponential behavior in 3 out of 5 galaxies of their sample, suggesting that this may be more common in $\mathrm{cD}$ galaxies than previously thought. A visual inspection of these galaxies reveals that they have high ellipticities. We calculated an average outer ellipticity $e=0.32 \pm 0.09$, which is slightly higher than the average found for double profile BCGs (see the next section).

In general terms we have also noticed that the inner components of the double profile BCGs have effective radii $r_{e} \sim 1-10 \mathrm{kpc}$ and Sérsic indices $n \sim 1-6$, being the averages $r_{e}=5 \pm 4 \mathrm{kpc}$ and $n=3.7 \pm 1.5$ respectively. This values are quite similar to those reported by Gonzalez et al. (2003) in their preliminary paper for a sample of 31 BCGs. However, these authors use a different approach to fit the luminosity profiles. They use two Sérsic functions instead our Sérsic + exponential approach.

\subsection{Single Profile BCGs versus Double Profile BCGs}

A question that arises after our analysis is to establish if single profile BCGs actually differ in morphology from double profile BCGs. The models of the light profiles we apply do not imply conjectures on galaxy morphologies. Are BCGs with single and double profile of a different nature? If so, is this difference environmental or intrinsic?

In order to answer these questions we carried out a series of tests in which we explored BCGs properties together with the global cluster properties. One of them is the Kormendy relation (KR; Kormendy 1977) which is presented in Figure 3. This is an empirical scaling relation between surface brightness $\mu_{e}$ and effective radius $r_{e}$ and it represents the projection of the Fundamental Plane (Djorgovski \& Davies 1987). For the case of single profile BCGs both $\mu_{e}$ and $r_{e}$ are directly obtained from the fitting profile. However, in the case of double profile BCGs, we calculate these parameters from the double profile, i.e., using the sum of the Sérsic and exponential profiles. A linear regression applied to the whole sample gives

$$
\mu_{e}=18.72( \pm 0.06)+3.13( \pm 0.05) \log \left(r_{e} / \mathrm{kpc}\right)
$$

The slope of the KR obtained for all BCGs is $a_{B C G}=3.13 \pm 0.05$ which is similar to the value obtained by Oegerle \& Hoesel (1991) for a sample of 43 BCGs (i.e., $a_{B C G}=3.12 \pm 0.14$ ).

However, Bildfell et al. (2008) obtain for a sample of BCGs selected from 48 X-ray luminous clus- 
ters $\left(a_{B C G}=3.44 \pm 0.13\right)$, which is considerably steeper than our value and than the value of "normal" ellipticals $a_{\text {ellip }}=3.02 \pm 0.14$ (Oegerle \& Hoesel, 1991). Nevertheless, when we apply the same analysis to single profile and double profile BCGs separately we obtain for single profile BCGs

$$
\mu_{e}=18.65( \pm 0.07)+3.29( \pm 0.06) \log \left(r_{e} / \mathrm{kpc}\right)
$$

and for double profile BCGs

$$
\mu_{e}=19.03( \pm 0.10)+2.79( \pm 0.08) \log \left(r_{e} / \mathrm{kpc}\right)
$$

Different slopes suggest that the formation timescale of the single profile BCGs could differ from their double profile counterpart (von der Linden et al. 2007).

It is interesting to note that these values are calculated integrating the galaxy luminosity profiles up to infinity. If we consider a finite radius instead, total luminosities will change and therefore both $r_{e}$ and $\mu_{e}$ will also change. We then have also calculated the KR for both subsamples considering different galaxy radii $(r=100,200,300 \mathrm{kpc})$. We observed that the slope of the KR flattens for smaller radii and tends to a similar value for both subsamples $(a \sim 2.6$ at $r=100 \mathrm{kpc})$. This effect could easily explain the differences found in the size-luminosity relation between von der Linden et al. (2007) and Lauer et al. (2007) and Bernardi et al. (2007). von der Linden (2007) defines the $r_{e}$ by integrating luminosity profiles up to the $\mu=23$ mag arcsec $^{-2}$ isophote, while Lauer et al. (2007) and Bernardi et al. (2007) integrate the luminosity profiles up to infinity.

We also find that single profile BCGs show a median total absolute magnitude $M_{T \text {, single }}=$ $-23.8 \pm 0.7$, while double profile BCGs have $M_{T, \text { double }}=-24.0 \pm 0.5$. The KolmogorovSmirnov (K-S) test applied to these data indicates that the $M_{T}$ distributions for single profile and double profile BCGs are statistically different at $99.4 \%$ confidence level. Figure 4 shows the total absolute magnitude distributions for single and double profile BCGs.

To verify our findings and to rule out a possible dependence on our fitting models, we calculated the total luminosity within different diaphragms with radius ranging from 5 to $70 \mathrm{kpc}$. We find that integrated luminosities for both subsamples are indistinguishable up to $r=15 \mathrm{kpc}$. This can be seen in Figure 5 where we plot the absolute integrated magnitude versus the radius of the circular diaphragm expressed in kpc. The vertical line indicates $r=14.5 \mathrm{kpc}$ where metric luminosity and alpha parameter are calculated. Average integrated luminosities beyond $20 \mathrm{kpc}$ are $\sim 0.2 \mathrm{mag}$ brighter for double profile BCGs. We have applied the K-S test to both subsamples and results indicate that they do not statistically differ for $r=5$, 10 , and $14.5 \mathrm{kpc}$, while for larger radii the integrated luminosity distribution are truly different at the $99 \%$ confidence level. We highlight that $r=20 \mathrm{kpc}$ is close to value for which the Sérsic component equals the exponential component (see the case for A0690 in Figure 2). From the data tabulated in Table 2, it is straightforward to compute the radius where $I_{\text {Sersic }}=I_{\text {exp }}$ for each of the double profile BCGs. Averaging these values we find $\langle r\rangle=13 \pm 5 \mathrm{kpc}$ at $\langle\mu\rangle=22.5 \pm 0.7$ mag arcsec $^{-2}$. In other words, this result corroborates that the extra-light observed in double profile $\mathrm{BCGs}$ originates in the intermediate regions of the galaxies and not in the inner regions. The same conclusion can also be derived from Figure 6 where we plotted the total sum of the luminosity profiles corresponding to the single and double profile BCGs. Prior to the sum, individual profiles are normalized to their effective radii. Note that the extra-light of the double profile BCGs becomes apparent in the region $1<r / r_{e}<5$, which roughly correspond to $\sim 15-75 \mathrm{kpc}$.

\subsection{Ellipticities and Isophote Twisting}

We have also explored other photometric parameters which are not directly related to the surface brightness profile fitting functions. Figure 7 shows the ellipticity distributions for single and double profile BCGs, and Figure 8 shows the absolute value of the inner minus outer ellipticity distributions for the same subsamples. Outer ellipticities are measured at $\mu \sim 23-24 \mathrm{mag}$ $\operatorname{arcsec}^{-2}$ which is approximately half a magnitude brighter than our limit surface magnitudes. Similarly, the inner ellipticity is measured at $r \sim 4-5^{\prime \prime}$, which corresponds to 3-4 times the average seeing. In Figure 7 we show that double profile BCGs have higher ellipticities that single profile BCGs. We obtain an average ellipticity < $e_{\text {double }}>=0.30 \pm 0.10$ for double profile BCGs and $<e_{\text {single }}>=0.26 \pm 0.11$ for single profile BCGs. A 
K-S test applied to these data indicates that the ellipticity distributions for single and double profile BCGs are statistically different at a $98.3 \%$ confidence level. Similar results are obtained for the inner minus outer ellipticity of these subsamples. We obtain an average $\left\langle\Delta e_{\text {double }}\right\rangle=0.15 \pm 0.10$ for the double profile BCGs, while for single profile BCGs we have $\left\langle\Delta e_{\text {single }}>=0.10 \pm 0.09\right.$. Again, the K-S test indicates different distributions at $99.9 \%$ confidence level. The logarithmic slope of the metric luminosity (calculated at $r=14.5 \mathrm{kpc}) \alpha$ is also higher in double profile BCGs $\left(<\alpha_{\text {double }}>=0.65 \pm 0.12\right)$ than in single profile BCGs $\left(<\alpha_{\text {single }}>=0.59 \pm 0.14\right)$. Figure 9 presents the distributions of $\alpha$ for single and double profile BCGs respectively. A K-S test applied to these data establishes that the distributions are statistically different at a $99.9 \%$ confidence level.

The presence of isophote twisting was also explored. We calculated the outer minus inner position angle of the isophotes for those galaxies with ellipticities greater than 0.15 since position angle errors associated with rounder isophotes are large. We found similar values for both single and double profile BCGs $\left(<\Delta p a>=8^{\circ} \pm 9^{\circ}\right)$.

\subsection{Sérsic + Exponential or Exponential + Sérsic?}

By combining a large set of hydrodynamical simulations spanning a broad range of luminosity profiles of various masses, Hopkins et al. (2009) show an alternative way to separate luminosity profiles into an inner starburst component and outer pre-starburst component for "cusp" ellipticals which are formed via gas-rich mergers. These authors show that dissipational mergers give rise to two-component luminosity profiles which can be accounted by an exponential function (inner component) plus a Sérsic model (outer component). The exponential function accounts for the extra-light that was formed in a compact central starburst and makes the inner light profile of the galaxy deviate from a single Sérsic in the galaxy core. The outer component was formed by violent relaxation of the stars already present in the precursor galaxies.

We fitted to our double component BCGs an exponential (inner) + Sérsic (outer) model in the order proposed by Hopkins et al. (2009). As an example, we show the results of this fitting for the
BCG A0690 in Figure 10. The agreement between model and measurements is excellent and the Hopkins model properly accounts for the luminosity profile. We also compared the rms and $\chi^{2}$ values with those obtained with our original Sérsic + exponential model and we find that both models are equally good. In other words, our approach and the Hopkins model are, from a mathematical point of view, equivalent.

The results of fitting exponential + Sérsic models to our double profile galaxies are summarized in Table 3, which lists the same 10 parameters as in Table 2. In this case Sérsic parameters $\mu_{e}, r_{e}$ and $n$ correspond to the outer component, while the exponential $\mu_{0}$ and $r_{0}$ parameters correspond to the inner component. Obtained $\chi^{2}$ values indicate that this fit proved to be equally good as the inner Sérsic + outer exponential form. However, for some galaxies we inexorably obtained unrealistic $(\geq 300 \mathrm{kpc})$ effective radius using the Hopkins model, this is not the case for the Sérsic + exponential approach. Given that our results suggest that the extra-light comes from the intermediate regions (see also the next section), we believe that the Sérsic + exponential profile fitting is the appropriate selection for the BCGs analized in the present work.

\subsection{Extra-light and D-cD Envelopes}

Around $\sim 20 \%$ of giant elliptical galaxies have extensive, low-luminosity envelopes. These galaxies are known as D type, and those with the largest envelopes are denominated cD galaxies (Mackie, 1992). The envelopes, which are seen as deviations from the de Vaucouleurs profile, are quite faint, occur below $24 \mathrm{mag} \operatorname{arcsec}^{-2}$ in the $V$ band, and they extend well beyond $100 \mathrm{kpc}$ in projected size. Thus, only few giant ellipticals have confirmed envelopes.

We explore if the extra-light found at intermediate radii is related to an eventual $\mathrm{cD}$ envelope. From the works of Kemp et al. (2007), Seigar et al. (2007), Mackie (1992), and Schombert (1986, 1987, 1988), we have found 24 BCGs, cataloged as cD galaxies with confirmed envelopes, in common with our sample. These are: A85, A150, A151, A193, A262, A358, A539, A779, A1177, A1238, A1767, A1795, A1809, A1904, A1913, A2028, A2147, A2162, A2199, A2366, A2572, A2589, A2634, and A2670. Table 2 shows that 19 (79\%) of 
these 24 galaxies are double profile BCGs, while 5 $(21 \%)$ are single profile BCGs. Note that three galaxies (A151, A1767, and A2589) from those five single profile BCGs, have $r_{e}>100 \mathrm{kpc}$, and $n>6$. These parameters are very close (see Section 4) to the limit $\left(r_{e}>300 \mathrm{kpc}, n>8\right)$ where it is necessary to include an additional exponential profile to obtain a reasonable fit. On the other hand, one must also note that a visual classification as cD, does not necessarily imply the presence of an envelope. Schombert (1986), and Seigar et al. (2007) cataloged A496, A505, A1691, A2029, A2052, A2107, A2197, and A2666, as cDs without envelopes. For all those galaxies, except A2197, our surface profile modeling is consistent with just one component.

These results strongly suggest that the extralight found in double component BCGs at intermediate radii is related to the faint envelope. Moreover, they indicate that this component is not only confined to the outskirt of the parent galaxy. Galaxy halos generally considered an outer component in galaxy structure appear to originate in the inner regions of BCGs.

\subsection{BCGs Morphologies and IR Emission}

Although a visual inspection of our BCGs suggests that they all are early-type galaxies, we probe the possibility that the differences we observe in the profiles are due to actual differences in the morphology. The Galaxy Zoo project (http://zoo1.galaxyzoo.org/) provides the morphological types for a large sample of galaxies observed by the Sloan Digital Sky Survey (SDSS; Lintott et al. 2010). In this catalog 66 of the BCGs studied here have a morphological classification: $31(47 \%)$ are single profile BCGs, while $35(53 \%)$ are double profile BCGs. The Lintott et al. (2010) catalog gives the probability that a particular galaxy is an early-type galaxy or latetype (spiral) galaxy. We find that both single and double profile BCGs have the same probability to be classified as elliptical galaxies in the Galaxy Zoo catalog. However, from the 31 single profile BCGs with morphological classification only $5(16 \%)$ belong to the 'unknown' category, while for the 35 double profile BCGs in the catalog this number rises up to $9(26 \%)$. It is also interesting to note that a visual inspection of these nine double profile BCGs with unknown morphology are mostly interacting galaxies with two or three near companions, while the five single profile BCGs with unkown classification appear to us as normal ellipticals.

We have scrutinized the infrared emission of a subsample of our BCGs using data from Quillen et al. (2008). They report on an imaging survey with the SpitzerSpaceTelescope of 62 BCGs with optical line emission. We have 12 BCGs in common with Quillen et al. (2008), 6 having single component luminosity profiles and 6 having double component luminosity profiles. Analysis of the 24-8 $\mu \mathrm{m}$ flux ratios shows that only one $(17 \%)$ of single profile BCG (A2052) have infrared excess, while for double profile BCGs this number increases to $4(67 \%)$. Infrared excess is a star formation signature. In fact, O'Dea et al. (2010) in their study of seven BCGs using HubbleSpaceTelescope(HST) ultra-violet and Spitzer infrared data found that all these galaxies have extended UV continuum and $L-\alpha$ emission as well as an infrared excess. Based on their findings O'Dea et al. (2010) confirm that the BCGs they study are actively forming stars. Moreover, they suggest that the IR excess is indeed associated with star formation and they also confirm that the FUV continuum emission extends over a region $\sim 7-28 \mathrm{kpc}$. Although these results are only for a few BCGs in our sample, they suggest that the "extra-light" observed in the double profile BCGs indicates active star formation in the intermediate regions of these galaxies.

\subsection{Global Cluster Properties}

In this section we compare BCGs properties to those of the host cluster such as cluster X-ray luminosity, the projected distance of the BCG with respect to the center of the $\mathrm{X}$-ray emission and the BCG position angle with respect to that of the cluster. We have used data taken from Ledlow et al. (2003) to determine the offset in kpc between the X-ray peak and the optical position of the BCG. X-ray cluster luminosities were taken from Sadat et al. (2004) data, while the position angle of the clusters are obtained from Plionis (1994), Rhee \& Katgert (1987), and Binggeli (1982). In this case we have only selected those clusters with ellipticities $>0.15$, since for smaller ellipticities position angles have large errors. 
Single and double profile BCGs have similar orientations relative to the whole cluster and X-ray luminosity distributions. We did not observe any difference between the single profile and double profile BCG samples with respect to the cluster position angle and X-ray cluster luminosity. However, Bildfell et al. (2008) report that brighter BCGs are located closer to the X-ray peak emission. In our case, considering that on average double profile BCGs are brighter than single profile BCGs we should observe larger offsets in single profile BCGs. Nevertheless, we find no significant differences between single profile and double profile BCGs offsets with respect to the X-ray center emission of the cluster. Moreover, we do not find any correlation between the total absolute magnitude and X-ray offset. Figure 11 shows that there is no obvious trend between the total absolute magnitude of both single profile and double profile BCGs.

\section{Conclusions}

We have established the existence of two subpopulations of BCGs based on their luminosity profiles. We analyze a uniquely large sample of 430 BCGs and find that $48 \%$ of these galaxies have a light profile that deviates from the standard single Sérsic model. The luminosity profiles of these galaxies are in fact better described by a double component model consisting of an inner Sérsic profile and an outer exponential component. The necessity of an outer exponential component conveys the presence of extra-light at intermediate radii corresponding to surface magnitudes $\sim 22$ magarcsec $^{-2}$. We have found strong evidence from a subsample of 24 BCGs that extralight is closely related to the presence of a faint envelope. Similarly, from other subsample of 12 BGCs we also found evidence that links extra-light and star formation.

This work highlights the need to cover a large spatial scale when deriving the structural parameters of large galaxies. Accurate parameters can only be obtained when the entire galaxy is considered and this often requires the creation of composite light profiles using data from different telescopes as clearly illustrated by Kormendy et al. (2009). HST detectors provide a superb spatial resolution that has been fundamental for the study of the deviation from the Sérsic law of the light profile in the inner regions of galaxies i.e. cusps and evacuated cores (Ferrarese et al. 2006). However, HST detectors do not provide the field of view necessary to truthfully derive the structural parameters of galaxies with a light profile that deviates from a single Sérsic law at large radii.

\section{Acknowledgments}

We are thankful to M. Postman (STScI) for giving us access to the data used in this study. We also wish to thank the anonymous referee for his useful comments which helped to clarify and strengthen this paper. This research has made use of the NASA Astrophysics Data System Bibliographic services (ADS) and the NASA/IPAC Extragalactic Database (NED) which is operated by the Jet Propulsion Laboratory, California Institute of Technology, under contract with the National Aeronautics and Space Administration. This work has been partially supported with grants from Consejo Nacional de Investigaciones Científicas y Técnicas de la República Argentina (CONICET), Secretaría de Ciencia y Tecnología de la Universidad de Córdoba and Ministerio de Ciencia y Tecnología de Córdoba, Argentina.

Bernardi, M., Hyde, J.B., Sheth, R.K., Miller, C.J., Nichol, R.C. 2007, AJ, 133, 1741

Bildfell, C.; Hoekstra, H.; Babul, A.; Mahadavi, A. 2008, MNRAS, 389, 1637

Binggeli, B. 1982, A\&A, 107, 338

Caon, N., Capaccioli, M., \& D'Onofrio, M. 1993, MNRAS, 265, 1013

Coenda, V., Donzelli, C.J., Muriel, H., Quintana, H., \& Infante, L. 2005, AJ, 129, 1237

Conroy, C., Wechsler, R. H., \& Kravtsov, A. V. 2007, ApJ, 668, 826

de Lucia, G. \& Blaizot, J. 2007, MNRAS, 375, 2

de Vaucouleurs, G. 1948, Ann. Astrophys., 11, 247 
Djorgovski, S., \& Davis, M. 1987, ApJ, 313, 59

Donzelli, C.J., Chiaberge, M., Macchetto, F. D., Madrid, J.P., Capetti, A., \& Marchesini, D. 2007, ApJ, 667, 780

Ferrarese, L., et al. 2006, ApJS, 164, 334

Freeman, K. C. 1970, ApJ, 160, 811

Gonzalez, A. H., Zabludoff, A. I., \& Zaritsky, D. 2003, Ap\&SS, 285, 67

Gonzalez, A. H., Zabludoff, A. I., \& Zaritsky, D. 2005, ApJ, 618, 195

Graham, A.W., \& Driver, S.P. 2005, PASA, 22, 118

Graham, A.W., Lauer, T.R., Colless, M. \& Postman, M. 1996, ApJ, 465, 534

Hopkins, P.F., Cox, T. J., Dutta, S. N., Hernquist, Lars., Kormendy, J., Lauer, T. R. 2009, ApJS, 181, 135

Jedrzejewski, R. 1987, MNRAS, 226, 747

Kent, S.M. 1985, ApJS, 59, 115

Kent, S.N., Guzmán Jiménez, V., Ramírez Beraud, P., Hernández Ibarra, F.J., \& Pérez Grana, J.A. 2007, in IAU Symp. 235, Galaxy Evolution Across the Hubble Time, ed. F. Combes \& J. Palous (Cambridge: Cambridge Univ. Press), 213

Kormendy, J. 1977, ApJ, 218, 333

Kormendy, J., Fisher, D. B., Cornell, M. E., \& Bender, R. 2009, ApJS, 182, 216

La Barbera, F., Busarello, G., Merluzzi, P., De La Rosa, I., Coppola, G., \& Haynes, C.P. 2008, PASP, 120, 681

Landolt, A.U. 1983, AJ, 88, 853
Lauer, T. R., \& Postman, M. 1992, ApJ, 400, L50

Lauer, T.R., et al. 2007, ApJ, 662, 808

Ledlow, M.J., Voges, W., Owen, F.N., \& Burns, J.O. 2003, AJ, 126, 2740

Lintott, C. et al. 2011, MNRAS, 410, 166

Lugger, P.M. 1984, ApJ, 286, 106

Mackie, G. 1992, ApJ, 400, 65

Oegerle, W.R.; Hoessel, J.G. 1991, ApJ, 375, 150

O’Dea, K. et al. 2010, ApJ, 719, 1619

Plionis, M. 1994, ApJS, 95, 401

Postman, M. \& Lauer, T. R. 1995, ApJ, 440, 28

Quillen, A.C. et al. 2008, ApJS, 176, 39

Rhee, G., \& Katgert, P. 1987, A\&A, 183, 217

Sadat, R., Blanchard, A., Kneib, J.P., Mathez, G., Madore, B., \& Mazzarella, J.M. 2004, A\&A, 424, 1097

Seigar, M.S., Graham, A.W., Jerjen, H. 2007, MNRAS, 378, 1575

Schombert, J.M. 1986, ApJS, 60, 603

Schombert, J.M. 1987, ApJS, 64, 643

Schombert, J.M. 1988, ApJ, 328, 475

Schombert, J.M. \& Bothun, G.D. 1987, AJ, 93, 60

Sérsic, J.L. 1963, Boletin de la Asociación Argentina de Astronomía, 6, 41

Sérsic, J.L. 1968, Atlas de Galaxias Australes 
(Córdoba: Obs. Astronóm.)

von der Linden, A., Best, P.N., Kauffmann, G., \& White, S. 2007, MNRAS, 379, 867

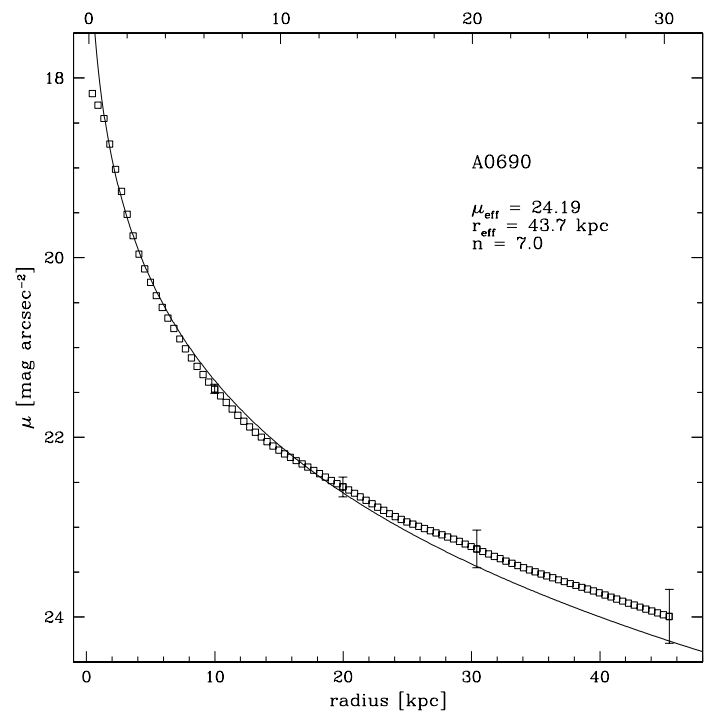

Fig. 1.- BCG A0690 luminosity profile with the single Sérsic fitting. Upper scale is in arcsec.



Fig. 2.- Inner Sérsic (short dashed line) + outer exponential (long dashed line) fitting model for A0690 luminosity profile. 




Fig. 3.- Kormedy relation for sample galaxies. Black dots represent single profile BCGs, while red dots represent double profile BCGs. Best fits for both subsamples are also shown.



Fig. 4.- Total magnitude distributions for single (black line) and double profile (red line) BCGs.

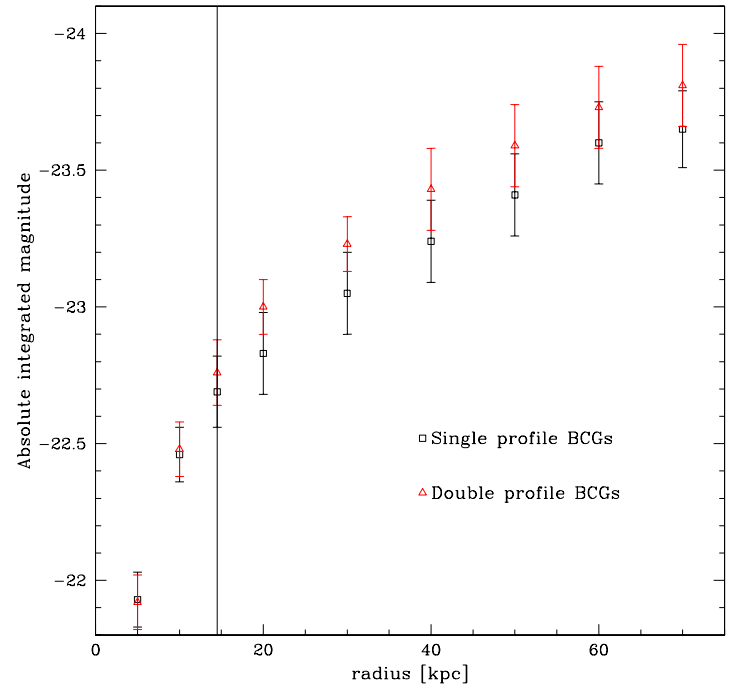

Fig. 5.- Integrated absolute magnitudes vs. diaphragm radius in kpc. The vertical line indicates where alpha parameter and metric magnitudes are calculated.

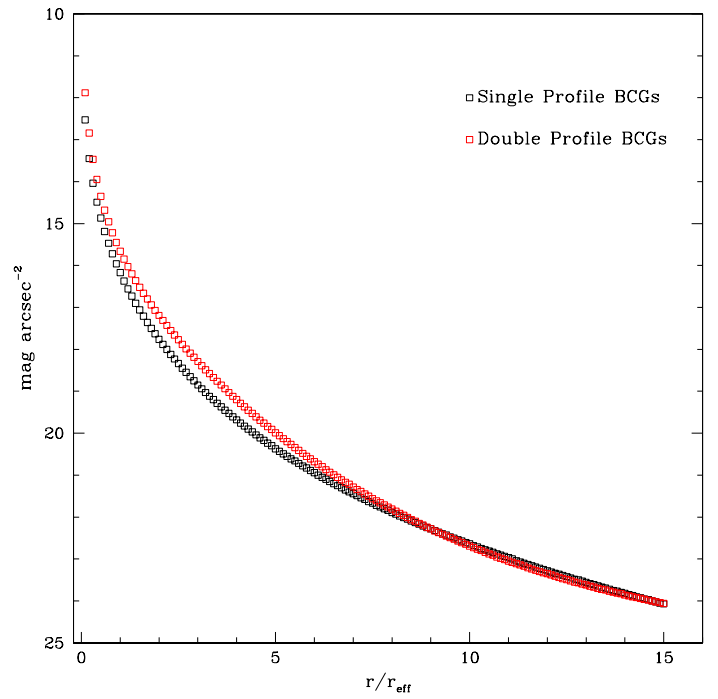

Fig. 6.- Luminosity profiles obtained using the stacking technique for all single and double profile BCGs. Prior to the stacking the individual profiles were normalized at the effective radius. 


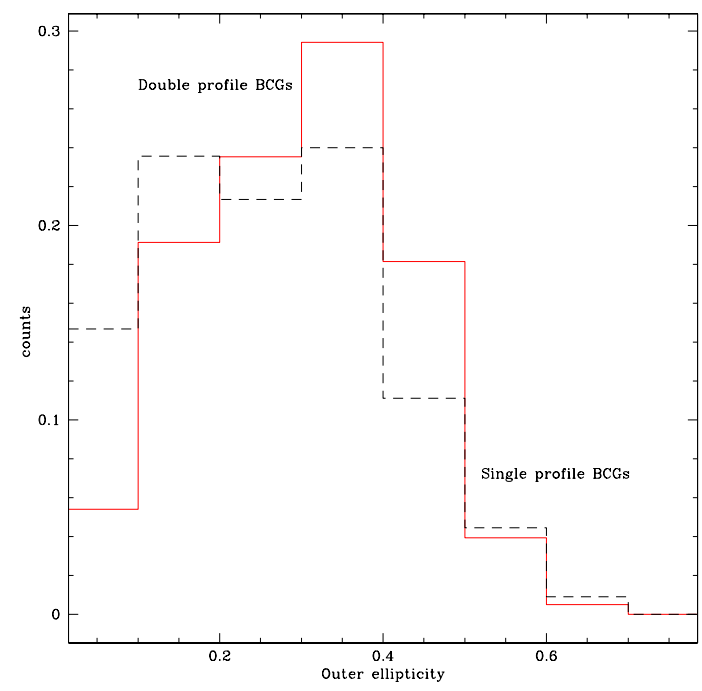

Fig. 7.- Outer ellipticity distributions for single (black line) and double profile (red line) BCGs.

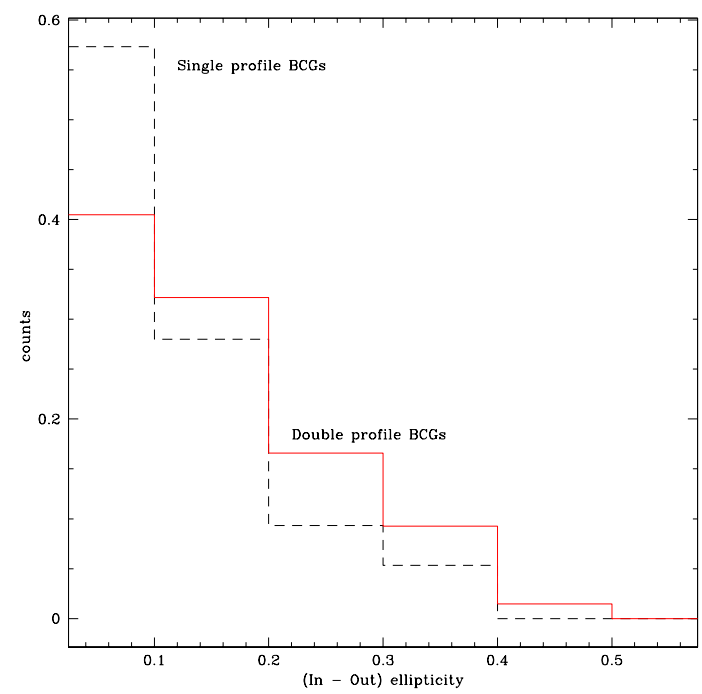

Fig. 8.- Outer minus inner ellipticity distributions for single (black line) and double profile (red line) BCGs.

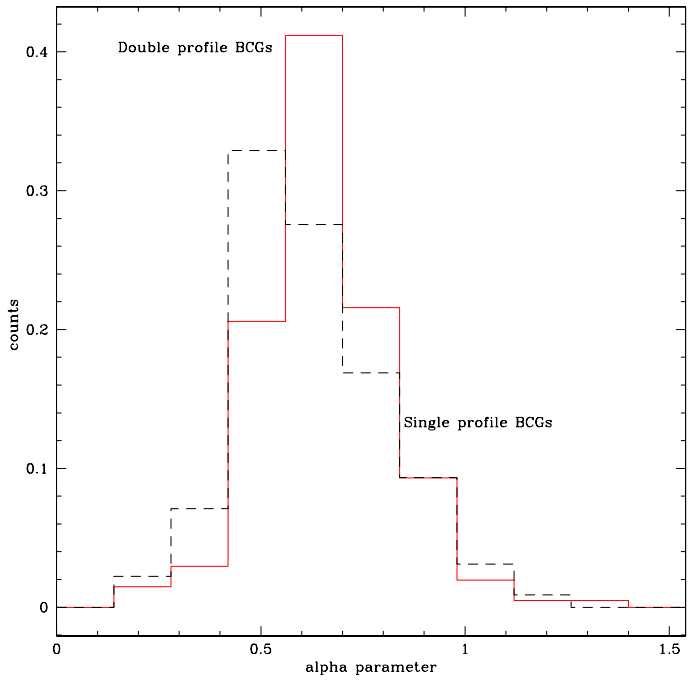

Fig. 9.- $\alpha$ parameter distributions for single (black line) and double profile (red line) BCGs.



Fig. 10. - Exp + Sérsic model fit for A0690 luminosity profile. Outer Sérsic (short dashed line) and inner exponential (long dashed lined) can be observed. 


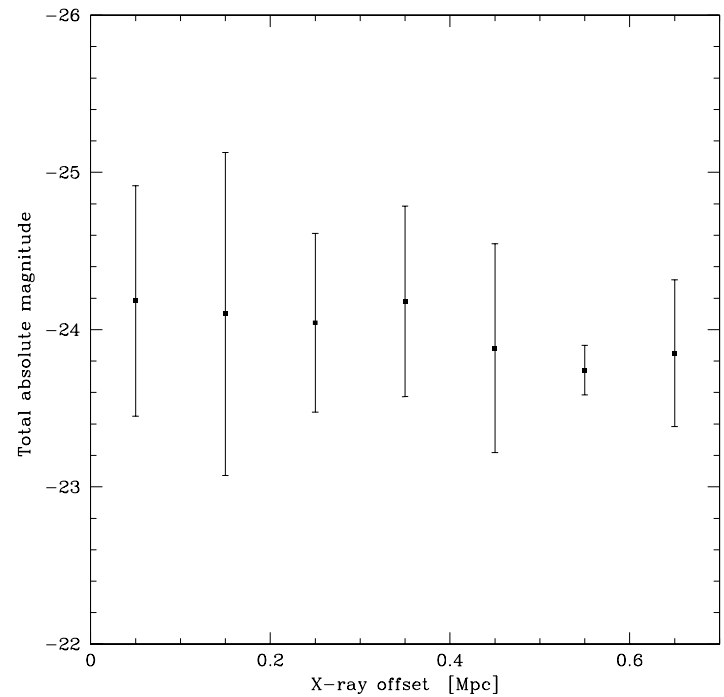

Fig. 11.- BCGs total absolute magnitude vs. $\mathrm{X}$-ray offset for the whole BCG sample. 
TABle 1

BCGs Imaging Runs

\begin{tabular}{rcccc}
\hline \hline Run & Date & Observatory & Pixel Scale (") & FOV (') \\
\hline 1 & 1989 Oct & CTIO & 0.273 & 3.6 \\
2 & 1989 Nov & KPNO 4m & 0.299 & 4.0 \\
3 & $1990 \mathrm{Nov}$ & CTIO & 0.273 & 3.6 \\
4 & $1991 \mathrm{Mar}$ & KPNO 2.1m & 0.304 & 5.2 \\
5 & $1991 \mathrm{Apr}$ & CTIO & 0.434 & 7.4 \\
6 & 1993 Sep & KPNO 2.1m & 0.304 & 5.2 \\
7 & 1993 Nov & CTIO & 0.434 & 7.4 \\
8 & $1994 \mathrm{May}$ & KPNO 2.1m & 0.304 & 5.2 \\
9 & 1994 May & CTIO & 0.434 & 7.4 \\
10 & 1994 Oct & KPNO 2.1m & 0.304 & 5.2 \\
11 & $1994 \mathrm{Dec}$ & CTIO & 0.434 & 7.4 \\
12 & $1995 \mathrm{Apr}$ & CTIO & 0.434 & 7.4 \\
13 & $1995 \mathrm{Apr}$ & KPNO 2.1m & 0.304 & 5.2 \\
\hline
\end{tabular}


TABLE 2

BCGs Photometrical Parameters

\begin{tabular}{|c|c|c|c|c|c|c|c|c|c|c|c|c|c|c|c|}
\hline $\begin{array}{c}\text { Name } \\
(1)\end{array}$ & $\begin{array}{l}\mu_{e} \\
(2)\end{array}$ & $\begin{array}{l}r_{e} \\
(3)\end{array}$ & $\begin{array}{c}n \\
(4)\end{array}$ & $\begin{array}{l}\mu_{0} \\
(5)\end{array}$ & $\begin{array}{l}r_{0} \\
(6)\end{array}$ & $\begin{array}{c}M_{\text {Sersic }} \\
\quad(7)\end{array}$ & $\begin{array}{c}M_{\exp } \\
(8)\end{array}$ & $\begin{array}{c}M_{T} \\
(9)\end{array}$ & $\begin{array}{l}S / e \\
(10)\end{array}$ & $\begin{array}{c}\alpha \\
(11)\end{array}$ & $\begin{array}{l}e_{i n} \\
(12)\end{array}$ & $\begin{array}{l}e_{\text {out }} \\
(13)\end{array}$ & $\begin{array}{c}p a_{i n} \\
(14)\end{array}$ & $\begin{array}{c}p a_{\text {out }} \\
(15)\end{array}$ & $\begin{array}{c}M_{\text {metric }} \\
\quad(16)\end{array}$ \\
\hline A0014 & 26.65 & 387.58 & 5.08 & - & - & -26.65 & 0.00 & -26.65 & - & 1.10 & 0.35 & 0.56 & 56.5 & 52.0 & -22.87 \\
\hline $\mathrm{A} 0027$ & 24.41 & 32.51 & 7.58 & - & - & -23.67 & 0.00 & -23.67 & - & 0.59 & 0.24 & 0.28 & -54.0 & -61.2 & -22.18 \\
\hline $\mathrm{A} 0071$ & 20.73 & 5.18 & 5.49 & - & - & -23.28 & 0.00 & -23.28 & - & 0.49 & 0.06 & 0.05 & 51.1 & 31.1 & -22.45 \\
\hline $\mathrm{A} 0074$ & 23.24 & 23.04 & 6.45 & - & - & -24.05 & 0.00 & -24.05 & - & 0.54 & 0.28 & 0.30 & 42.2 & 39.6 & -22.93 \\
\hline $\mathrm{A} 0075$ & 21.86 & 10.58 & 4.74 & - & - & -23.55 & 0.00 & -23.55 & - & 0.55 & 0.09 & 0.23 & 85.0 & 60.4 & -22.74 \\
\hline A0076 & 24.08 & 34.80 & 7.58 & - & - & -24.09 & 0.00 & -24.09 & - & 0.57 & 0.05 & 0.17 & -66.4 & -118.7 & -22.80 \\
\hline $\mathrm{A} 0077$ & 26.39 & 190.00 & 6.94 & - & - & -25.65 & 0.00 & -25.65 & - & 0.91 & 0.13 & 0.51 & -62.5 & -64.0 & -23.04 \\
\hline A0080 & 21.59 & 5.92 & 2.24 & - & - & -22.29 & 0.00 & -22.29 & - & 0.46 & 0.11 & 0.06 & -64.2 & -71.0 & -21.98 \\
\hline A0085 & 21.43 & 12.43 & 0.86 & 21.63 & 26.00 & -23.47 & -24.24 & -24.68 & 0.49 & 1.30 & 0.11 & 0.35 & -27.3 & -32.1 & -23.18 \\
\hline A0086 & 22.55 & 14.89 & 6.17 & - & - & -23.76 & 0.00 & -23.76 & - & 0.56 & 0.26 & 0.17 & 70.6 & 74.8 & -22.90 \\
\hline A0102 & 23.73 & 32.22 & 7.41 & - & - & -24.36 & 0.00 & -24.36 & - & 0.61 & 0.13 & 0.11 & -64.2 & -41.3 & -22.97 \\
\hline $\mathrm{A} 0114$ & 22.73 & 20.87 & 5.85 & - & - & -24.26 & 0.00 & -24.26 & - & 0.59 & 0.16 & 0.28 & 72.9 & 73.6 & -23.01 \\
\hline A0116 & 21.44 & 9.44 & 4.52 & - & - & -23.74 & 0.00 & -23.74 & - & 0.58 & 0.17 & 0.17 & 5.5 & 15.5 & -22.93 \\
\hline $\mathrm{A} 0117$ & 24.06 & 47.47 & 6.06 & - & - & -24.73 & 0.00 & -24.73 & - & 0.76 & 0.12 & 0.28 & -7.7 & -19.9 & -23.20 \\
\hline A0119 & 26.71 & 247.61 & 7.87 & - & - & -25.77 & 0.00 & -25.77 & - & 0.83 & 0.12 & 0.33 & 34.8 & 35.9 & -23.01 \\
\hline A0126 & 20.74 & 3.10 & 3.61 & - & - & -21.78 & 0.00 & -21.78 & - & 0.26 & 0.04 & 0.09 & -38.6 & 38.9 & -21.55 \\
\hline A0133 & 21.10 & 7.64 & 1.11 & 21.46 & 23.80 & -22.87 & -24.23 & -24.50 & 0.29 & 0.93 & 0.07 & 0.43 & 33.7 & 22.4 & -22.95 \\
\hline $\mathrm{A} 0134$ & 21.19 & 5.86 & 4.72 & - & - & -22.98 & 0.00 & -22.98 & - & 0.47 & 0.14 & 0.05 & 10.5 & 1.6 & -22.40 \\
\hline $\mathrm{A} 0147$ & 21.75 & 10.17 & 6.06 & - & - & -23.64 & 0.00 & -23.64 & - & 0.49 & 0.16 & 0.09 & 33.6 & 31.6 & -22.83 \\
\hline $\mathrm{A} 0150$ & 20.93 & 6.34 & 1.91 & 22.01 & 26.20 & -22.91 & -23.89 & -24.26 & 0.40 & 0.75 & 0.11 & 0.43 & -2.9 & -4.8 & -22.81 \\
\hline A0151 & 25.86 & 146.25 & 8.06 & - & - & -25.51 & 0.00 & -25.51 & - & 0.78 & 0.17 & 0.26 & 73.5 & 76.9 & -23.11 \\
\hline A0152 & 18.92 & 1.38 & 5.65 & 20.93 & 11.80 & -22.24 & -23.32 & -23.66 & 0.37 & 0.84 & 0.04 & 0.28 & 39.0 & 17.2 & -22.64 \\
\hline A0154 & 21.82 & 11.13 & 5.21 & 22.33 & 28.00 & -23.76 & -23.72 & -24.50 & 1.04 & 0.66 & 0.22 & 0.39 & -38.9 & -44.1 & -23.05 \\
\hline A0158 & 19.06 & 1.66 & 4.15 & 20.97 & 10.20 & -22.28 & -22.90 & -23.39 & 0.56 & 0.71 & 0.13 & 0.12 & -59.4 & -48.6 & -22.58 \\
\hline A0160 & 20.38 & 3.04 & 1.04 & 21.05 & 12.50 & -21.49 & -23.18 & -23.38 & 0.21 & 0.78 & 0.03 & 0.24 & -73.1 & -92.1 & -22.44 \\
\hline A0161 & 20.53 & 3.65 & 4.74 & 22.33 & 23.50 & -22.64 & -23.40 & -23.84 & 0.50 & 0.67 & 0.05 & 0.31 & 4.9 & -42.9 & -22.07 \\
\hline A0168 & 24.51 & 48.01 & 7.41 & - & - & -24.38 & 0.00 & -24.38 & - & 0.65 & 0.06 & 0.25 & -25.6 & -26.5 & -22.83 \\
\hline $\mathrm{A} 0171$ & 22.75 & 17.23 & 6.49 & - & - & -24.04 & 0.00 & -24.04 & - & 0.63 & 0.14 & 0.12 & 86.5 & 30.7 & -23.00 \\
\hline $\mathrm{A} 0174$ & 20.44 & 3.71 & 0.98 & 21.56 & 13.02 & -21.97 & -22.89 & -23.27 & 0.43 & 0.66 & 0.02 & 0.16 & 5.9 & -64.9 & -22.53 \\
\hline A0179 & 21.14 & 5.27 & 3.73 & 21.92 & 16.30 & -22.62 & -22.93 & -23.54 & 0.75 & 0.63 & 0.22 & 0.38 & -27.3 & -25.6 & -22.35 \\
\hline A0189 & 21.13 & 7.32 & 3.06 & - & - & -23.15 & 0.00 & -23.15 & - & 0.40 & 0.42 & 0.50 & -2.8 & -6.7 & -22.24 \\
\hline A0193 & 18.60 & 1.78 & 4.13 & 21.52 & 22.00 & -22.84 & -23.97 & -24.30 & 0.36 & 0.59 & 0.04 & 0.23 & -69.9 & -73.3 & -22.88 \\
\hline A0194 & 18.59 & 1.30 & 1.53 & 19.95 & 7.70 & -21.51 & -23.12 & -23.34 & 0.23 & 0.68 & 0.25 & 0.36 & 56.6 & 60.5 & -22.58 \\
\hline A0195 & 21.62 & 8.16 & 6.58 & - & - & -23.42 & 0.00 & -23.42 & - & 0.50 & 0.09 & 0.05 & -84.7 & 11.2 & -22.69 \\
\hline A0208 & 21.66 & 7.95 & 5.32 & 22.36 & 26.80 & -23.37 & -23.76 & -24.34 & 0.70 & 0.67 & 0.06 & 0.33 & 10.7 & 25.5 & -22.95 \\
\hline $\mathrm{A} 0225$ & 25.24 & 84.64 & 7.09 & - & - & -24.95 & 0.00 & -24.95 & - & 0.78 & 0.15 & 0.27 & 81.2 & 81.6 & -22.92 \\
\hline A0240 & 24.61 & 55.91 & 5.38 & - & - & -24.50 & 0.00 & -24.50 & - & 0.86 & 0.09 & 0.21 & 4.6 & 9.3 & -22.74 \\
\hline A0245 & 21.95 & 8.70 & 3.28 & - & - & -23.02 & 0.00 & -23.02 & - & 0.57 & 0.21 & 0.27 & -65.2 & -81.4 & -22.30 \\
\hline A0246 & 23.73 & 24.12 & 6.33 & - & - & -23.70 & 0.00 & -23.70 & - & 0.62 & 0.16 & 0.39 & 17.8 & 11.0 & -22.42 \\
\hline A 0257 & 19.86 & 2.99 & 4.26 & 21.13 & 11.90 & -22.91 & -23.21 & -23.83 & 0.75 & 0.64 & 0.26 & 0.42 & 9.9 & 24.1 & -22.76 \\
\hline A0260 & 20.95 & 6.17 & 4.50 & 21.85 & 20.90 & -23.17 & -23.46 & -24.08 & 0.76 & 0.62 & 0.12 & 0.33 & 46.3 & 73.2 & -22.89 \\
\hline A0261 & 20.19 & 4.08 & 2.60 & 21.69 & 15.30 & -22.79 & -23.00 & -23.65 & 0.83 & 0.57 & 0.10 & 0.18 & -2.7 & -9.8 & -22.81 \\
\hline A0262 & 20.33 & 3.18 & 1.75 & 21.16 & 16.60 & -21.54 & -23.33 & -23.52 & 0.19 & 0.76 & 0.07 & 0.41 & -73.6 & 42.0 & -22.21 \\
\hline $\mathrm{A} 0267$ & 25.90 & 105.21 & 6.29 & - & - & -24.66 & 0.00 & -24.66 & - & 0.90 & 0.19 & 0.33 & 78.4 & 72.0 & -22.58 \\
\hline A0268 & 20.23 & 2.85 & 3.70 & 22.80 & 14.30 & -22.31 & -21.89 & -22.88 & 1.47 & 0.47 & 0.14 & 0.27 & 2.7 & -11.5 & -22.06 \\
\hline A0279 & 22.81 & 18.86 & 5.29 & 22.56 & 30.60 & -24.01 & -23.76 & -24.65 & 1.25 & 0.81 & 0.11 & 0.24 & 40.2 & 47.6 & -23.10 \\
\hline A0292 & 23.37 & 45.49 & 3.11 & - & - & -25.01 & 0.00 & -25.01 & - & 0.88 & 0.31 & 0.58 & -84.9 & -85.6 & -22.94 \\
\hline A0295 & 21.47 & 4.64 & 2.99 & 21.44 & 17.30 & -21.84 & -23.48 & -23.70 & 0.22 & 0.66 & 0.11 & 0.44 & 76.4 & 67.0 & -22.76 \\
\hline A0311 & 22.79 & 15.71 & 5.43 & 22.36 & 38.00 & -23.59 & -24.38 & -24.81 & 0.48 & 0.80 & 0.17 & 0.53 & 15.7 & 29.0 & -22.83 \\
\hline A0326 & 21.06 & 5.91 & 3.14 & - & - & -22.87 & 0.00 & -22.87 & - & 0.38 & 0.42 & 0.11 & 21.5 & 87.7 & -22.22 \\
\hline A 0347 & 20.95 & 3.99 & 3.66 & - & - & -21.82 & 0.00 & -21.82 & - & 0.32 & 0.30 & 0.21 & -76.7 & -66.5 & -21.28 \\
\hline A0357 & 21.61 & 7.00 & 2.51 & - & - & -22.68 & 0.00 & -22.68 & - & 0.35 & 0.41 & 0.47 & -42.1 & -39.9 & -21.85 \\
\hline A0358 & 23.57 & 32.81 & 5.13 & - & - & -24.34 & 0.00 & -24.34 & - & 0.76 & 0.17 & 0.28 & 3.9 & 11.0 & -22.87 \\
\hline A0376 & 25.76 & 94.82 & 8.00 & - & - & -24.66 & 0.00 & -24.66 & - & 0.73 & 0.05 & 0.27 & -82.9 & 81.7 & -22.71 \\
\hline A0396 & 20.47 & 2.35 & 1.66 & - & - & -20.87 & 0.00 & -20.87 & - & 0.22 & 0.47 & 0.35 & 7.7 & 7.7 & -20.42 \\
\hline A0397 & 20.31 & 3.82 & 3.44 & 21.78 & 19.20 & -22.66 & -23.38 & -23.83 & 0.51 & 0.61 & 0.14 & 0.40 & 39.2 & 60.5 & -22.87 \\
\hline A0399 & 21.82 & 10.41 & 1.22 & 21.83 & 33.30 & -22.92 & -24.65 & -24.85 & 0.20 & 1.13 & 0.23 & 0.43 & 46.5 & 39.8 & -22.80 \\
\hline A0401 & 28.27 & 968.92 & 7.46 & - & - & -27.25 & 0.00 & -27.25 & - & 1.02 & 0.26 & 0.58 & 35.8 & 24.7 & -23.01 \\
\hline
\end{tabular}


TABLE 2-Continued

\begin{tabular}{|c|c|c|c|c|c|c|c|c|c|c|c|c|c|c|c|}
\hline $\begin{array}{l}\text { Name } \\
(1)\end{array}$ & $\begin{array}{l}\mu_{e} \\
(2)\end{array}$ & $\begin{array}{l}r_{e} \\
(3)\end{array}$ & $\begin{array}{c}n \\
(4)\end{array}$ & $\begin{array}{l}\mu_{0} \\
(5)\end{array}$ & $\begin{array}{l}r_{0} \\
(6)\end{array}$ & $\begin{array}{l}M_{\text {Sersic }} \\
\quad(7)\end{array}$ & $\begin{array}{c}M_{\text {exp }} \\
\quad(8)\end{array}$ & $\begin{array}{c}M_{T} \\
(9)\end{array}$ & $\begin{array}{l}S / e \\
(10)\end{array}$ & $\begin{array}{c}\alpha \\
(11)\end{array}$ & $\begin{array}{l}e_{i n} \\
(12)\end{array}$ & $\begin{array}{l}e_{\text {out }} \\
(13)\end{array}$ & $\begin{array}{l}p a_{i n} \\
(14)\end{array}$ & $\begin{array}{c}p a_{\text {out }} \\
(15)\end{array}$ & $\begin{array}{c}M_{\text {metric }} \\
(16)\end{array}$ \\
\hline A0404 & 20.33 & 4.04 & 1.09 & 21.26 & 12.40 & -22.27 & -23.03 & -23.47 & 0.50 & 0.62 & 0.27 & 0.37 & 22.4 & 24.7 & -22.57 \\
\hline A0415 & 22.20 & 9.26 & 3.88 & 21.95 & 26.00 & -22.91 & -24.02 & -24.36 & 0.36 & 0.86 & 0.12 & 0.32 & -20.1 & -12.5 & -22.72 \\
\hline A0419 & 21.68 & 7.33 & 4.55 & - & - & -22.85 & 0.00 & -22.85 & - & 0.40 & 0.28 & 0.32 & -61.2 & -25.0 & -22.06 \\
\hline A 0423 & 25.05 & 61.90 & 6.80 & - & - & -24.48 & 0.00 & -24.48 & - & 0.70 & 0.19 & 0.47 & -75.5 & -70.3 & -22.56 \\
\hline A 0428 & 18.82 & 1.65 & 3.51 & 21.71 & 6.80 & -22.43 & -21.29 & -22.75 & 2.86 & 0.41 & 0.03 & 0.09 & 8.0 & 48.6 & -22.18 \\
\hline A0450 & 22.01 & 8.26 & 4.35 & - & - & -22.92 & 0.00 & -22.92 & - & 0.49 & 0.21 & 0.16 & 12.5 & -2.2 & -22.25 \\
\hline A0496 & 25.74 & 101.00 & 5.62 & - & - & -24.57 & 0.00 & -24.57 & - & 0.78 & 0.16 & 0.37 & -8.6 & -3.9 & -22.75 \\
\hline A0497 & 20.95 & 2.41 & 3.58 & 21.51 & 9.70 & -21.31 & -22.44 & -22.77 & 0.35 & 0.81 & 0.20 & 0.28 & 26.5 & 31.0 & -21.95 \\
\hline A0500 & 20.90 & 5.92 & 2.90 & 22.44 & 30.00 & -23.03 & -23.79 & -24.23 & 0.50 & 0.63 & 0.21 & 0.37 & 9.0 & 33.0 & -22.83 \\
\hline A0505 & 24.64 & 78.09 & 6.80 & - & - & -25.29 & 0.00 & -25.29 & - & 0.84 & 0.14 & 0.17 & -51.9 & 11.0 & -23.39 \\
\hline A0514 & 23.13 & 21.69 & 5.56 & - & - & -23.98 & 0.00 & -23.98 & - & 0.61 & 0.19 & 0.31 & -53.3 & -49.9 & -22.90 \\
\hline A0533 & 22.59 & 16.32 & 4.98 & - & - & -23.75 & 0.00 & -23.75 & - & 0.55 & 0.24 & 0.17 & -61.2 & -44.8 & -22.69 \\
\hline A0536 & 25.23 & 84.14 & 6.54 & - & - & -25.15 & 0.00 & -25.15 & - & 0.84 & 0.29 & 0.41 & -34.1 & -29.2 & -23.05 \\
\hline A0539 & 21.03 & 5.56 & 4.76 & 22.31 & 22.30 & -22.91 & -23.15 & -23.79 & 0.80 & 0.50 & 0.04 & 0.36 & -11.3 & -2.0 & -22.59 \\
\hline A0543 & 19.33 & 2.39 & 2.48 & - & - & -22.63 & 0.00 & -22.63 & - & 0.27 & 0.07 & 0.02 & -64.1 & -47.6 & -22.06 \\
\hline A0548 & 21.41 & 7.07 & 4.08 & 20.39 & 6.60 & -22.98 & -22.45 & -23.50 & 1.63 & 0.64 & 0.34 & 0.14 & 64.4 & 82.7 & -22.73 \\
\hline A0550 & 22.20 & 10.71 & 4.26 & 23.00 & 51.50 & -23.33 & -24.52 & -24.83 & 0.34 & 0.76 & 0.15 & 0.38 & -43.4 & -56.8 & -22.79 \\
\hline A0553 & 21.69 & 9.57 & 3.44 & 23.30 & 57.80 & -23.48 & -24.46 & -24.83 & 0.40 & 0.67 & 0.16 & 0.57 & -68.4 & -67.1 & -22.87 \\
\hline A0564 & 20.09 & 3.86 & 1.28 & 23.03 & 29.20 & -22.64 & -23.28 & -23.76 & 0.55 & 0.39 & 0.23 & 0.35 & 29.5 & 42.1 & -22.54 \\
\hline A0568 & 21.59 & 11.11 & 2.87 & - & - & -23.84 & 0.00 & -23.84 & - & 0.69 & 0.18 & 0.11 & 31.9 & -22.7 & -23.07 \\
\hline A0569 & 22.11 & 13.01 & 4.72 & - & - & -23.22 & 0.00 & -23.22 & - & 0.50 & 0.13 & 0.15 & -4.4 & 34.3 & -22.54 \\
\hline A0576 & 20.35 & 4.19 & 4.74 & - & - & -22.94 & 0.00 & -22.94 & - & 0.32 & 0.17 & 0.08 & -60.2 & 46.9 & -22.37 \\
\hline A0582 & 20.38 & 3.79 & 4.88 & 22.95 & 26.60 & -22.92 & -23.09 & -23.76 & 0.86 & 0.51 & 0.01 & 0.04 & -52.3 & 16.2 & -22.63 \\
\hline A0592 & 20.55 & 4.44 & 4.29 & 22.00 & 13.30 & -22.17 & -21.67 & -22.70 & 1.58 & 0.52 & 0.07 & 0.21 & 84.6 & -86.6 & -22.58 \\
\hline A0595 & 21.76 & 7.99 & 5.62 & - & - & -23.27 & 0.00 & -23.27 & - & 0.49 & 0.06 & 0.16 & 76.2 & 78.8 & -22.51 \\
\hline A0600 & 23.54 & 24.95 & 4.52 & - & - & -23.89 & 0.00 & -23.89 & - & 0.73 & 0.10 & 0.35 & 26.8 & -1.1 & -22.60 \\
\hline A0602 & 21.18 & 5.32 & 3.30 & - & - & -22.56 & 0.00 & -22.56 & - & 0.47 & 0.17 & 0.23 & 58.0 & 41.3 & -22.39 \\
\hline A0607 & 21.59 & 8.46 & 2.68 & - & - & -23.26 & 0.00 & -23.26 & - & 0.69 & 0.02 & 0.07 & -60.2 & 31.1 & -22.81 \\
\hline A0612 & 23.12 & 30.92 & 5.13 & - & - & -24.96 & 0.00 & -24.96 & - & 0.96 & 0.06 & 0.01 & -81.9 & 4.2 & -23.54 \\
\hline A0634 & 21.72 & 10.91 & 3.07 & - & - & -23.22 & 0.00 & -23.22 & - & 0.50 & 0.17 & 0.14 & -77.2 & 67.5 & -22.46 \\
\hline A0644 & 24.11 & 29.04 & 5.59 & 22.34 & 31.40 & -23.64 & -24.01 & -24.59 & 0.71 & 0.85 & 0.23 & 0.49 & 7.4 & 10.4 & -22.55 \\
\hline A0671 & 20.14 & 4.99 & 2.21 & 21.33 & 23.30 & -23.23 & -24.29 & -24.64 & 0.38 & 0.73 & 0.21 & 0.27 & 23.7 & 30.7 & -23.20 \\
\hline A0690 & 21.65 & 9.54 & 5.24 & 22.54 & 28.30 & -23.72 & -23.65 & -24.44 & 1.06 & 0.66 & 0.10 & 0.20 & 41.7 & 53.0 & -23.06 \\
\hline A0695 & 22.59 & 17.78 & 3.75 & - & - & -23.90 & 0.00 & -23.90 & - & 0.70 & 0.23 & 0.29 & -53.4 & -54.3 & -22.79 \\
\hline A0744 & 24.10 & 40.05 & 6.33 & - & - & -24.43 & 0.00 & -24.43 & - & 0.76 & 0.03 & 0.06 & 71.6 & -49.6 & -22.86 \\
\hline A0757 & 20.80 & 4.44 & 2.17 & 23.23 & 23.50 & -22.31 & -22.42 & -23.12 & 0.90 & 0.49 & 0.10 & 0.12 & 26.2 & 69.8 & -22.23 \\
\hline A0779 & 20.70 & 7.99 & 3.72 & 21.60 & 27.20 & -23.57 & -23.97 & -24.54 & 0.69 & 0.58 & 0.16 & 0.41 & $\begin{array}{l}-23.7 \\
\end{array}$ & -21.0 & -23.10 \\
\hline A0780 & 21.94 & 10.39 & 3.70 & 22.30 & 26.25 & -23.30 & -23.60 & -24.21 & 0.76 & 0.86 & 0.12 & 0.32 & -36.5 & -34.6 & -22.85 \\
\hline A0819 & 24.42 & 57.38 & 6.85 & - & - & -24.94 & 0.00 & -24.94 & - & 0.71 & 0.25 & 0.45 & -22.9 & -27.4 & -22.98 \\
\hline A0834 & 17.97 & 0.68 & 2.87 & 19.55 & 4.20 & -21.21 & -22.38 & -22.70 & 0.34 & 0.47 & 0.36 & 0.27 & -10.2 & -5.7 & -22.17 \\
\hline A0838 & 24.18 & 30.16 & 7.04 & - & 1.20 & -23.70 & 0.00 & -23.70 & - & 0.62 & 0.11 & 0.27 & 6.5 & 86.0 & -22.39 \\
\hline A0841 & 21.15 & 7.34 & 4.35 & 22.88 & 33.50 & -23.48 & -23.61 & -24.30 & 0.89 & 0.63 & 0.09 & 0.30 & -29.8 & -28.1 & -22.95 \\
\hline A0865 & 21.03 & 3.79 & 1.17 & - & - & -21.53 & 0.00 & -21.53 & - & 0.23 & 0.45 & 0.51 & -31.6 & -29.9 & -21.08 \\
\hline A0912 & 22.18 & 9.14 & 5.75 & - & - & -23.03 & 0.00 & -23.03 & - & 0.43 & 0.11 & 0.13 & 68.6 & -9.1 & -22.29 \\
\hline A0930 & 19.48 & 1.93 & 4.03 & 22.16 & 14.80 & -22.14 & -22.50 & -23.09 & 0.72 & 0.58 & 0.08 & 0.18 & -27.0 & -8.2 & -22.07 \\
\hline A0957 & 22.10 & 14.38 & 3.89 & 22.90 & 25.20 & -23.89 & -23.92 & -24.66 & 0.97 & 0.86 & 0.20 & 0.22 & -81.9 & 57.3 & -23.00 \\
\hline A0970 & 21.81 & 6.50 & 5.24 & 23.01 & 23.90 & -22.61 & -22.70 & -23.41 & 0.92 & 0.61 & 0.10 & 0.16 & -54.6 & -61.3 & -22.19 \\
\hline A0978 & 20.74 & 5.67 & 5.21 & 21.91 & 23.70 & -23.36 & -23.76 & -24.33 & 0.69 & 0.64 & 0.08 & 0.24 & 7.7 & -18.1 & -22.99 \\
\hline A0979 & 21.87 & 9.13 & 4.59 & 22.63 & 20.10 & -23.19 & -22.68 & -23.71 & 1.60 & 0.65 & 0.07 & 0.11 & -50.0 & -39.3 & -22.71 \\
\hline A0993 & 22.54 & 12.29 & 6.41 & - & - & -23.35 & 0.00 & -23.35 & - & 0.58 & 0.06 & 0.06 & 52.8 & 1.8 & -23.05 \\
\hline A0994 & 22.61 & 18.69 & 4.90 & - & - & -23.98 & 0.00 & -23.98 & - & 0.54 & 0.35 & 0.41 & -21.3 & -18.6 & -22.70 \\
\hline A0999 & 20.89 & 6.10 & 5.08 & 23.28 & 33.50 & -23.15 & -22.94 & -23.80 & 1.22 & 0.44 & 0.19 & 0.24 & -9.1 & -29.4 & -22.55 \\
\hline A1003 & 19.43 & 1.90 & 2.58 & 20.89 & 11.80 & -21.96 & -23.30 & -23.58 & 0.29 & 0.70 & 0.06 & 0.29 & -31.5 & -53.8 & -22.65 \\
\hline A1016 & 19.77 & 2.90 & 3.89 & 22.23 & 13.50 & -22.52 & -22.02 & -23.05 & 1.59 & 0.43 & 0.10 & 0.14 & 9.6 & 0.1 & -22.35 \\
\hline A 1020 & 20.27 & 3.93 & 5.41 & 22.73 & 18.60 & -23.10 & -22.46 & -23.58 & 1.79 & 0.53 & 0.05 & 0.16 & -46.6 & -41.8 & -22.76 \\
\hline A1021 & 20.33 & 4.44 & 1.58 & 21.67 & 15.70 & -22.82 & -23.31 & -23.84 & 0.64 & 0.64 & 0.17 & 0.25 & -88.3 & -89.8 & -22.92 \\
\hline A1032 & 21.19 & 7.93 & 3.86 & - & - & -23.52 & 0.00 & -23.52 & - & 0.50 & 0.36 & 0.32 & 31.5 & 25.1 & -22.59 \\
\hline A1035 & 23.46 & 28.91 & 5.38 & - & - & -24.33 & 0.00 & -24.33 & - & 0.73 & 0.06 & 0.15 & -9.4 & $\begin{array}{l}20.1 \\
-14.6\end{array}$ & -22.99 \\
\hline
\end{tabular}


TABLE 2-Continued

\begin{tabular}{|c|c|c|c|c|c|c|c|c|c|c|c|c|c|c|c|}
\hline $\begin{array}{l}\text { Name } \\
(1)\end{array}$ & $\begin{array}{l}\mu_{e} \\
(2)\end{array}$ & $\begin{array}{l}r_{e} \\
(3)\end{array}$ & $\begin{array}{c}n \\
(4)\end{array}$ & $\begin{array}{l}\mu_{0} \\
(5)\end{array}$ & $\begin{array}{l}r_{0} \\
(6)\end{array}$ & $\begin{array}{c}M_{\text {Sersic }} \\
\quad(7)\end{array}$ & $\begin{array}{c}M_{\exp } \\
(8)\end{array}$ & $\begin{array}{c}M_{T} \\
(9)\end{array}$ & $\begin{array}{l}S / e \\
(10)\end{array}$ & $\begin{array}{c}\alpha \\
(11)\end{array}$ & $\begin{array}{l}e_{i n} \\
(12)\end{array}$ & $\begin{array}{l}e_{\text {out }} \\
(13)\end{array}$ & $\begin{array}{c}p a_{i n} \\
(14)\end{array}$ & $\begin{array}{c}p a_{\text {out }} \\
(15)\end{array}$ & $\begin{array}{l}M_{\text {metric }} \\
\quad(16)\end{array}$ \\
\hline A1066 & 20.73 & 6.52 & 2.26 & 23.01 & 47.10 & -23.31 & -24.22 & -24.61 & 0.43 & 0.72 & 0.18 & 0.30 & -40.4 & -60.3 & -22.84 \\
\hline A1069 & 21.28 & 6.61 & 5.03 & 21.20 & 16.30 & -23.18 & -23.71 & -24.23 & 0.62 & 0.78 & 0.28 & 0.12 & -86.8 & -85.6 & -22.98 \\
\hline A 1100 & 20.49 & 4.99 & 2.33 & 21.97 & 20.90 & -22.88 & -23.39 & -23.92 & 0.62 & 0.55 & 0.19 & 0.38 & -10.0 & -17.5 & -22.74 \\
\hline A1139 & 21.39 & 6.74 & 4.83 & 22.45 & 22.60 & -22.92 & -22.99 & -23.71 & 0.93 & 0.57 & 0.06 & 0.32 & -51.2 & -82.0 & -22.55 \\
\hline A 1142 & 22.65 & 15.08 & 6.67 & 23.46 & 49.00 & -23.51 & -23.60 & -24.31 & 0.92 & 0.54 & 0.15 & 0.24 & 36.6 & 51.1 & -22.55 \\
\hline A1145 & 21.49 & 6.31 & 3.56 & - & - & -22.70 & 0.00 & -22.70 & - & 0.44 & 0.17 & 0.16 & -44.3 & -50.1 & -22.12 \\
\hline A1149 & 19.97 & 3.11 & 3.95 & 21.58 & 16.30 & -22.74 & -23.35 & -23.84 & 0.57 & 0.65 & 0.11 & 0.33 & 68.8 & 69.7 & -22.76 \\
\hline A1169 & 22.08 & 9.21 & 6.41 & - & - & -23.21 & 0.00 & -23.21 & - & 0.48 & 0.22 & 0.28 & -32.0 & -75.3 & -22.25 \\
\hline A 1171 & 20.03 & 3.54 & 2.62 & 22.42 & 22.00 & -22.78 & -23.18 & -23.75 & 0.69 & 0.63 & 0.30 & 0.49 & 44.6 & 43.7 & -22.26 \\
\hline A 1177 & 23.13 & 20.88 & 6.41 & 22.29 & 29.00 & -23.70 & -23.62 & -24.41 & 1.08 & 0.71 & 0.13 & 0.42 & 47.5 & 39.8 & -22.77 \\
\hline A1185 & 21.45 & 10.10 & 1.87 & 23.94 & 95.70 & -23.21 & -24.60 & -24.87 & 0.28 & 0.66 & 0.12 & 0.07 & 39.5 & -15.0 & -22.87 \\
\hline A1187 & 20.80 & 5.69 & 2.93 & 21.80 & 16.70 & -23.14 & -23.25 & -23.95 & 0.91 & 0.64 & 0.18 & 0.21 & -81.6 & -65.2 & -22.95 \\
\hline A1190 & 23.57 & 21.29 & 4.74 & 21.85 & 20.50 & -23.48 & -23.63 & -24.31 & 0.87 & 0.95 & 0.28 & 0.32 & -7.6 & -12.4 & -22.72 \\
\hline A1203 & 21.58 & 9.28 & 2.54 & 23.43 & 39.10 & -23.33 & -23.44 & -24.14 & 0.90 & 0.75 & 0.16 & 0.17 & -47.1 & -25.8 & -22.81 \\
\hline A1213 & 20.93 & 7.84 & 2.42 & - & - & -23.41 & 0.00 & -23.41 & - & 0.50 & 0.27 & 0.09 & 66.5 & 88.5 & -22.75 \\
\hline A1216 & 19.77 & 2.69 & 1.85 & 21.22 & 9.80 & -22.18 & -22.54 & -23.12 & 0.72 & 0.52 & 0.22 & 0.30 & -50.2 & -46.5 & -22.38 \\
\hline A1228 & 19.39 & 2.34 & 4.18 & 21.81 & 12.10 & -22.52 & -22.25 & -23.14 & 1.28 & 0.43 & 0.06 & 0.19 & 13.8 & -42.3 & -22.42 \\
\hline A1238 & 21.00 & 4.64 & 4.67 & 22.15 & 22.50 & -22.67 & -23.48 & -23.90 & 0.47 & 0.68 & 0.08 & 0.43 & -79.9 & -77.7 & -22.56 \\
\hline A 1257 & 17.37 & 0.82 & 2.54 & 20.26 & 4.70 & -21.97 & -21.72 & -22.61 & 1.26 & 0.24 & 0.10 & 0.13 & -46.5 & -43.0 & -22.10 \\
\hline A 1267 & 21.28 & 7.21 & 4.42 & - & - & -23.05 & 0.00 & -23.05 & - & 0.33 & 0.23 & 0.30 & 44.9 & 53.4 & -22.43 \\
\hline A 1270 & 20.34 & 6.21 & 1.59 & - & - & -23.44 & 0.00 & -23.44 & - & 0.48 & 0.43 & 0.20 & -46.1 & -45.3 & -22.76 \\
\hline A 1279 & 24.32 & 38.01 & 6.80 & - & - & -24.07 & 0.00 & -24.07 & - & 0.63 & 0.17 & 0.31 & 59.4 & 55.8 & -22.56 \\
\hline A1308 & 21.99 & 9.41 & 4.13 & 23.29 & 34.60 & -23.09 & -23.20 & -23.90 & 0.90 & 0.55 & 0.10 & 0.12 & -26.0 & -38.3 & -22.61 \\
\hline A1314 & 19.76 & 3.67 & 2.54 & 21.67 & 23.70 & -22.84 & -23.82 & -24.19 & 0.41 & 0.57 & 0.19 & 0.36 & 86.8 & -86.2 & -22.81 \\
\hline A1317 & 21.93 & 10.26 & 4.98 & 22.52 & 51.90 & -23.50 & -24.92 & -25.18 & 0.27 & 0.73 & 0.18 & 0.57 & 38.9 & 55.4 & -22.89 \\
\hline A1318 & 23.96 & 35.95 & 5.38 & - & - & -24.18 & 0.00 & -24.18 & -2 & 0.54 & 0.22 & 0.29 & -8.8 & $\begin{array}{l}-7.6 \\
\end{array}$ & -22.75 \\
\hline A1334 & 21.66 & 8.47 & 5.05 & - & - & -23.32 & 0.00 & -23.32 & - & 0.51 & 0.21 & 0.22 & 88.8 & 77.4 & -22.53 \\
\hline A1344 & 24.04 & 52.80 & 5.08 & - & - & -24.98 & 0.00 & -24.98 & - & 0.72 & 0.39 & 0.32 & 80.3 & 78.8 & -23.05 \\
\hline A1365 & 22.79 & 15.86 & 6.62 & - & - & -23.80 & 0.00 & -23.80 & - & 0.50 & 0.21 & 0.22 & -56.0 & -62.1 & -22.67 \\
\hline A1367 & 23.00 & 25.76 & 5.56 & - & - & -23.97 & 0.00 & -23.97 & - & 0.56 & 0.13 & 0.24 & 4.1 & -10.8 & -22.74 \\
\hline A1371 & 21.74 & 7.06 & 5.88 & 22.66 & 19.90 & -22.99 & -22.72 & -23.62 & 1.28 & 0.65 & 0.08 & 0.19 & 59.6 & 44.0 & -22.50 \\
\hline A 1377 & 21.90 & 10.47 & 4.98 & - & - & -23.50 & 0.00 & -23.50 & - & 0.54 & 0.23 & 0.10 & 59.1 & 69.5 & -22.66 \\
\hline A 1400 & 23.35 & 22.75 & 3.34 & - & - & -23.66 & 0.00 & -23.66 & - & 0.81 & 0.16 & 0.23 & -61.3 & -65.9 & -22.45 \\
\hline A 1424 & 19.58 & 3.02 & $\begin{array}{l}5.04 \\
4.27\end{array}$ & 20.60 & 9.90 & -23.17 & -23.30 & -23.99 & 0.89 & 0.63 & 0.25 & 0.15 & 72.4 & 70.3 & -23.12 \\
\hline A1436 & 20.96 & 5.57 & 5.49 & - & - & -23.21 & 0.00 & -23.21 & - & 0.44 & 0.10 & 0.10 & -79.8 & -65.4 & -22.56 \\
\hline A1452 & 23.87 & 34.05 & 6.85 & - & - & -24.34 & 0.00 & -24.34 & - & 0.66 & 0.08 & 0.12 & 48.0 & 52.9 & -22.80 \\
\hline A 1474 & 19.71 & 1.74 & 1.10 & 19.63 & 7.40 & -21.20 & -23.67 & -23.78 & 0.10 & 0.67 & 0.51 & 0.58 & -12.1 & -10.0 & -22.57 \\
\hline A1507 & 20.54 & 4.45 & 3.45 & 21.48 & 15.60 & -22.88 & -23.34 & -23.89 & 0.65 & 0.70 & 0.20 & 0.29 & 48.0 & 44.1 & -22.82 \\
\hline A1520 & 22.35 & 16.82 & 5.85 & 22.29 & 34.30 & -24.23 & -24.25 & -24.99 & 0.98 & 0.75 & 0.16 & 0.33 & -17.7 & -32.1 & -23.26 \\
\hline A 1526 & 22.00 & 9.05 & 5.92 & - & - & -23.29 & 0.00 & -23.29 & - & 0.49 & 0.12 & 0.29 & -88.4 & -54.0 & -22.39 \\
\hline A1534 & 20.75 & 6.44 & 3.46 & 22.32 & 20.90 & -23.50 & -23.17 & -24.10 & 1.35 & 0.65 & 0.20 & 0.18 & -48.9 & -52.9 & -23.01 \\
\hline A1569 & 19.78 & 3.06 & 3.17 & 20.37 & 11.00 & -22.85 & $\begin{array}{l}-23.77 \\
\end{array}$ & -24.16 & 0.43 & 0.78 & 0.24 & 0.14 & -74.2 & -71.1 & -23.19 \\
\hline A1589 & 24.90 & 76.44 & 8.20 & - & - & -25.19 & 0.00 & -25.19 & - & 0.73 & 0.25 & 0.41 & 73.4 & 65.8 & -23.21 \\
\hline A1610 & 20.83 & 6.07 & 5.15 & 22.38 & 19.50 & -23.46 & -22.92 & -23.98 & 1.65 & 0.55 & 0.09 & 0.25 & -85.0 & -68.0 & -23.01 \\
\hline A1630 & 20.07 & 4.01 & 1.44 & 21.70 & 21.10 & -22.66 & -23.76 & -24.10 & 0.36 & 0.61 & 0.23 & 0.37 & 83.1 & 86.4 & -22.85 \\
\hline A1631 & 15.76 & 0.36 & 2.89 & 22.56 & 4.50 & -22.00 & -19.48 & -22.10 & 10.17 & 0.67 & 0.12 & 0.12 & 19.0 & 18.6 & -21.75 \\
\hline A1644 & 23.78 & 67.02 & 3.08 & - & - & -25.38 & 0.00 & -25.38 & - & 1.02 & 0.24 & 0.48 & 47.4 & 42.8 & -23.00 \\
\hline A 1648 & 20.47 & 4.95 & 0.96 & 21.81 & 24.20 & -22.58 & -24.01 & -24.27 & 0.27 & 0.52 & 0.32 & 0.41 & 59.4 & 55.1 & -22.78 \\
\hline A1691 & 22.55 & 22.89 & 2.99 & - & - & -24.39 & 0.00 & -24.39 & - & 0.92 & 0.18 & 0.07 & -34.3 & -14.1 & -23.16 \\
\hline A1709 & 23.08 & 17.18 & 6.80 & - & - & -23.57 & 0.00 & -23.57 & - & 0.55 & 0.08 & 0.16 & -53.0 & -20.1 & -22.63 \\
\hline A1736 & 23.34 & 39.24 & 6.21 & - & - & -25.00 & 0.00 & -25.00 & - & 0.58 & 0.33 & 0.43 & -44.4 & -45.1 & -23.26 \\
\hline A1741 & 21.56 & 7.88 & 6.58 & - & - & -23.49 & 0.00 & -23.49 & - & 0.48 & 0.16 & 0.11 & -34.9 & -39.2 & -22.69 \\
\hline A1749 & 20.41 & 5.81 & 3.27 & 22.04 & 22.40 & -23.53 & -23.54 & -24.29 & 0.99 & 0.59 & 0.25 & 0.22 & -85.8 & -76.0 & -23.10 \\
\hline A 1767 & 25.23 & 101.01 & 6.02 & - & - & -25.30 & 0.00 & -25.30 & - & 0.81 & 0.09 & 0.39 & -47.5 & -3.6 & -23.16 \\
\hline A 1773 & 21.22 & 7.12 & 5.62 & 21.18 & 27.70 & -23.52 & -24.93 & -25.19 & 0.27 & 0.65 & 0.27 & 0.07 & 30.0 & 12.5 & -22.82 \\
\hline A 1775 & 20.71 & 5.77 & 1.06 & 21.30 & 17.80 & -22.70 & -23.83 & -24.16 & 0.35 & 0.85 & 0.12 & 0.16 & -19.2 & -28.6 & -23.00 \\
\hline A 1780 & 22.04 & 13.08 & 3.40 & - & 11.00 & -23.75 & 0.00 & -23.75 & - & 0.64 & 0.29 & 0.30 & 83.4 & 75.8 & -22.70 \\
\hline A1795 & 20.94 & $\begin{array}{l}1.00 \\
6.02\end{array}$ & 1.88 & 21.70 & 27.50 & -22.83 & -24.37 & -24.60 & 0.24 & 0.81 & 0.10 & 0.34 & $\begin{array}{l}0.4 \\
9.5\end{array}$ & 15.4 & -22.94 \\
\hline
\end{tabular}


TABLE 2-Continued

\begin{tabular}{|c|c|c|c|c|c|c|c|c|c|c|c|c|c|c|c|}
\hline $\begin{array}{l}\text { Name } \\
(1)\end{array}$ & $\begin{array}{l}\mu_{e} \\
(2)\end{array}$ & $\begin{array}{l}r_{e} \\
(3)\end{array}$ & $\begin{array}{c}n \\
(4)\end{array}$ & $\begin{array}{l}\mu_{0} \\
(5)\end{array}$ & $\begin{array}{l}r_{0} \\
(6)\end{array}$ & $\begin{array}{l}M_{\text {Sersic }} \\
\quad(7)\end{array}$ & $\begin{array}{c}M_{\text {exp }} \\
\quad(8)\end{array}$ & $\begin{array}{c}M_{T} \\
(9)\end{array}$ & $\begin{array}{l}S / e \\
(10)\end{array}$ & $\begin{array}{c}\alpha \\
(11)\end{array}$ & $\begin{array}{l}e_{i n} \\
(12)\end{array}$ & $\begin{array}{l}e_{\text {out }} \\
(13)\end{array}$ & $\begin{array}{r}p a_{i n} \\
(14)\end{array}$ & $\begin{array}{c}p a_{o u t} \\
\quad(15)\end{array}$ & $\begin{array}{c}M_{\text {metric }} \\
\quad(16)\end{array}$ \\
\hline A 1800 & 22.88 & 45.78 & 2.38 & - & - & -25.46 & 0.00 & -25.46 & - & 1.09 & 0.39 & 0.57 & -18.6 & -22.2 & -23.23 \\
\hline A1809 & 21.78 & 12.38 & 4.93 & 22.81 & 45.50 & -24.07 & -24.37 & -24.98 & 0.76 & 0.69 & 0.18 & 0.33 & 64.7 & 54.7 & -23.26 \\
\hline A 1825 & 22.29 & 10.97 & 4.90 & 23.24 & 27.90 & -23.26 & -22.84 & -23.82 & 1.47 & 0.63 & 0.12 & 0.23 & -15.0 & -8.4 & -22.60 \\
\hline $\mathrm{A} 1827$ & 22.01 & 10.76 & 6.06 & - & - & -23.64 & 0.00 & -23.64 & - & 0.57 & 0.15 & 0.07 & 36.0 & 54.2 & -22.77 \\
\hline A 1828 & 21.04 & 7.22 & 2.53 & - & - & -23.28 & 0.00 & -23.28 & - & 0.44 & 0.36 & 0.37 & -33.6 & -28.7 & -22.53 \\
\hline A1831 & 22.75 & 18.26 & 4.98 & 21.80 & 28.20 & -23.99 & -24.37 & -24.95 & 0.70 & 0.86 & 0.10 & 0.41 & -20.0 & -29.8 & -23.23 \\
\hline A1836 & 23.22 & 26.32 & 5.24 & - & - & -24.14 & 0.00 & -24.14 & - & 0.60 & 0.09 & 0.29 & 48.6 & 37.8 & -22.91 \\
\hline A 1873 & 20.62 & 5.55 & 2.30 & 22.40 & 22.50 & -23.07 & -23.23 & -23.90 & 0.87 & 0.60 & 0.13 & 0.26 & -80.8 & -87.1 & -22.88 \\
\hline A 1890 & 23.78 & 49.81 & 5.18 & - & - & -25.08 & 0.00 & -25.08 & - & 0.76 & 0.21 & 0.34 & 40.6 & 42.9 & -23.26 \\
\hline A1898 & 23.37 & 18.80 & 6.13 & - & - & -23.54 & 0.00 & -23.54 & - & 0.60 & 0.11 & 0.17 & 1.9 & 21.2 & -22.44 \\
\hline A1899 & 21.50 & 9.54 & 2.83 & - & - & -23.41 & 0.00 & -23.41 & - & 0.58 & 0.36 & 0.33 & -43.1 & 82.3 & -22.59 \\
\hline A1904 & 21.91 & 12.00 & 4.76 & 21.85 & 22.30 & -23.88 & -23.80 & -24.59 & 1.08 & 0.76 & 0.17 & 0.35 & 20.9 & 44.4 & -23.18 \\
\hline A1913 & 21.99 & 10.73 & 6.02 & - & - & -23.55 & 0.00 & -23.55 & - & 0.56 & 0.04 & 0.00 & 24.3 & -72.1 & -22.76 \\
\hline A1964 & 19.74 & 2.78 & 5.29 & 22.10 & 23.00 & -22.89 & -23.58 & -24.04 & 0.53 & 0.57 & 0.16 & 0.10 & 14.6 & -24.2 & -22.52 \\
\hline A1982 & 20.94 & 5.80 & 3.09 & 21.44 & 16.10 & -22.96 & -23.42 & -23.96 & 0.65 & 0.67 & 0.30 & 0.40 & 48.3 & 46.9 & -22.72 \\
\hline A1983 & 21.89 & 11.28 & 6.29 & - & - & -23.76 & 0.00 & -23.76 & - & 0.34 & 0.26 & 0.29 & 28.4 & 26.9 & -22.59 \\
\hline A1991 & 26.26 & 201.64 & 6.41 & - & - & -25.74 & 0.00 & -25.74 & - & 0.90 & 0.18 & 0.36 & 15.6 & 9.7 & -22.86 \\
\hline A 2020 & 19.37 & 1.43 & 4.08 & 19.76 & 4.70 & -21.59 & -22.38 & -22.81 & 0.49 & 0.46 & 0.48 & 0.44 & -35.5 & -35.4 & -21.96 \\
\hline A 2022 & 19.94 & 3.62 & 3.55 & 21.98 & 22.70 & -22.99 & -23.61 & -24.10 & 0.57 & 0.65 & 0.12 & 0.12 & -34.2 & -56.8 & -22.88 \\
\hline A 2025 & 19.89 & 4.84 & 2.07 & 21.90 & 22.30 & -23.61 & -23.86 & -24.49 & 0.79 & 0.56 & 0.21 & 0.06 & -51.1 & -45.9 & -23.44 \\
\hline A 2028 & 21.57 & 10.27 & 3.32 & 22.78 & 35.40 & -23.68 & -23.86 & -24.52 & 0.84 & 0.71 & 0.20 & 0.32 & -81.1 & -81.4 & -23.05 \\
\hline A 2029 & 26.30 & 439.41 & 5.78 & - & - & -27.40 & 0.00 & -27.40 & - & 1.05 & 0.26 & 0.52 & 20.5 & 26.6 & -23.58 \\
\hline A 2040 & 20.57 & 3.67 & 5.21 & 21.94 & 26.20 & -22.54 & -23.90 & -24.17 & 0.28 & 0.72 & 0.12 & 0.44 & -55.2 & -55.8 & -22.43 \\
\hline A 2052 & 24.76 & 73.57 & 3.89 & - & - & -24.58 & 0.00 & -24.58 & - & 0.88 & 0.16 & 0.35 & 34.6 & 35.5 & -22.56 \\
\hline A 2055 & 21.19 & 5.15 & 3.80 & 21.86 & 21.00 & -22.51 & -23.52 & -23.88 & 0.39 & 0.42 & 0.25 & 0.35 & -26.1 & 27.1 & -21.89 \\
\hline A2063 & 24.15 & 44.25 & 4.27 & - & - & -24.21 & 0.00 & -24.21 & - & 0.80 & 0.04 & 0.38 & 39.0 & 29.1 & -22.71 \\
\hline A2065 & 23.87 & 27.62 & 7.30 & - & - & -23.90 & 0.00 & -23.90 & - & 0.67 & 0.18 & 0.09 & 2.9 & 11.1 & -22.51 \\
\hline A 2107 & 23.19 & 35.86 & 3.68 & - & - & -24.64 & 0.00 & -24.64 & - & 0.79 & 0.13 & 0.26 & -71.3 & -63.5 & -23.09 \\
\hline A 2143 & 21.06 & 5.41 & 6.21 & 21.87 & 15.10 & -23.20 & -22.99 & -23.85 & 1.21 & 0.72 & 0.06 & 0.08 & 21.1 & 16.3 & -22.84 \\
\hline A 2147 & 22.28 & 14.87 & 3.23 & 23.15 & 78.10 & -23.58 & -25.04 & -25.29 & 0.26 & 0.81 & 0.23 & 0.64 & 12.8 & 11.9 & -22.68 \\
\hline A 2152 & 21.86 & 9.98 & 6.45 & - & - & -23.53 & 0.00 & -23.53 & - & 0.48 & 0.07 & 0.08 & 16.4 & -70.5 & -22.68 \\
\hline A 2162 & 20.85 & 7.19 & 3.92 & 22.46 & 28.20 & -23.40 & -23.38 & -24.14 & 1.02 & 0.57 & 0.24 & 0.36 & 5.1 & 1.0 & -22.81 \\
\hline A 2184 & 21.65 & 7.05 & 3.19 & - & - & -22.66 & 0.00 & -22.66 & - & 0.49 & 0.15 & 0.26 & -15.0 & -17.7 & -22.17 \\
\hline A 2197 & 19.25 & 3.71 & 1.54 & 20.32 & 13.50 & -23.05 & -23.88 & -24.29 & 0.47 & 0.60 & 0.29 & 0.37 & -39.7 & -35.3 & -23.24 \\
\hline A 2198 & 21.42 & 5.64 & 5.00 & - & - & -22.66 & 0.00 & -22.66 & - & 0.42 & 0.12 & 0.20 & -8.2 & -3.6 & -21.98 \\
\hline A 2199 & 21.18 & 7.95 & 1.40 & 21.82 & 26.90 & -22.76 & -23.91 & -24.23 & 0.35 & 0.81 & 0.15 & 0.36 & 27.1 & 33.3 & -22.81 \\
\hline A 2247 & 21.13 & 6.67 & 2.19 & - & - & -22.73 & 0.00 & -22.73 & - & 0.41 & 0.10 & 0.23 & 27.2 & 3.4 & -22.34 \\
\hline A 2248 & 22.53 & 13.34 & 6.41 & - & - & -23.56 & 0.00 & -23.56 & - & 0.56 & 0.07 & 0.19 & 14.9 & 13.0 & -22.66 \\
\hline A2250 & 21.28 & 7.12 & 4.02 & - & - & -23.22 & 0.00 & -23.22 & - & 0.44 & 0.16 & 0.10 & -36.6 & -74.4 & -22.59 \\
\hline A 2256 & 20.84 & 8.91 & 1.87 & - & - & -23.73 & 0.00 & -23.73 & - & 0.52 & 0.35 & 0.30 & -80.6 & -76.1 & -22.97 \\
\hline A 2271 & 23.96 & 52.68 & 3.86 & - & - & -24.86 & 0.00 & -24.86 & - & 0.90 & 0.21 & 0.37 & -57.8 & -53.3 & -22.94 \\
\hline A 2293 & 23.19 & 19.05 & 5.99 & - & - & -23.68 & 0.00 & -23.68 & - & 0.73 & 0.26 & 0.33 & 76.8 & 72.4 & -22.61 \\
\hline A 2296 & 19.63 & 1.42 & 4.35 & 21.02 & 8.60 & -21.31 & -22.39 & -22.73 & 0.37 & 0.60 & 0.19 & 0.40 & 68.1 & 72.4 & -21.72 \\
\hline A 2308 & 24.51 & 44.68 & 6.37 & - & - & -24.28 & 0.00 & -24.28 & - & 0.74 & 0.07 & 0.26 & 38.5 & 17.9 & -22.73 \\
\hline A2309 & 20.02 & 3.49 & 4.18 & 22.17 & 17.80 & -22.88 & -22.86 & -23.62 & 1.02 & 0.57 & 0.21 & 0.22 & -61.2 & -68.0 & -22.67 \\
\hline A 2319 & 25.16 & 71.27 & 5.00 & 21.19 & 17.60 & -24.40 & -23.83 & -24.91 & 1.69 & 1.12 & 0.15 & 0.18 & 4.4 & 20.1 & -22.99 \\
\hline A 2331 & 22.07 & 6.98 & 5.18 & 21.67 & 17.50 & -22.57 & -23.44 & -23.84 & 0.45 & 0.86 & 0.06 & 0.27 & -86.3 & 89.4 & -22.59 \\
\hline A 2361 & 22.83 & 15.13 & 7.14 & - & - & -23.58 & 0.00 & -23.58 & - & 0.52 & 0.07 & 0.16 & 50.6 & 49.7 & -22.67 \\
\hline A 2362 & 22.52 & 15.75 & 6.13 & - & - & -23.91 & 0.00 & -23.91 & - & 0.55 & 0.15 & 0.09 & 68.6 & 85.0 & -23.01 \\
\hline A 2366 & 19.64 & 3.60 & 1.64 & 21.32 & 16.50 & -22.87 & -23.56 & -24.02 & 0.53 & 0.59 & 0.14 & 0.15 & -30.6 & -21.7 & -23.08 \\
\hline A 2372 & 20.16 & 4.35 & 1.55 & 21.97 & 17.40 & -22.75 & -23.05 & -23.66 & 0.76 & 0.58 & 0.13 & 0.29 & 7.2 & -4.4 & -22.81 \\
\hline A 2381 & 17.19 & 0.43 & 4.10 & 20.31 & 3.20 & -20.86 & -20.70 & -21.54 & 1.15 & 0.22 & 0.22 & 0.35 & 6.1 & 4.8 & -21.03 \\
\hline A 2382 & 20.41 & 3.97 & 1.55 & 21.31 & 16.30 & -22.33 & -23.59 & -23.89 & 0.31 & 0.82 & 0.17 & 0.10 & 8.6 & -67.8 & -22.76 \\
\hline A 2383 & 21.35 & 5.54 & 3.64 & - & - & -22.62 & 0.00 & -22.62 & - & 0.36 & 0.33 & 0.36 & -47.7 & -49.8 & -21.98 \\
\hline A 2388 & 25.06 & 57.29 & 6.71 & - & - & -24.22 & 0.00 & -24.22 & - & 0.68 & 0.13 & 0.44 & -80.5 & -75.2 & -22.46 \\
\hline A2399 & 21.75 & 12.37 & 3.91 & - & - & -23.91 & 0.00 & -23.91 & - & 0.62 & 0.32 & 0.21 & -77.3 & -70.1 & -22.92 \\
\hline A 2401 & 23.47 & 26.80 & 6.99 & - & - & -24.16 & 0.00 & -24.16 & - & 0.67 & 0.07 & 0.17 & -12.2 & -34.9 & -23.03 \\
\hline A 2412 & 20.33 & 4.55 & 1.79 & 23.43 & 90.80 & -22.81 & -25.23 & -25.34 & 0.11 & 0.57 & 0.15 & 0.11 & 31.0 & 22.6 & -22.65 \\
\hline
\end{tabular}


TABLE 2-Continued

\begin{tabular}{|c|c|c|c|c|c|c|c|c|c|c|c|c|c|c|c|}
\hline $\begin{array}{l}\text { Name } \\
(1)\end{array}$ & $\begin{array}{l}\mu_{e} \\
(2)\end{array}$ & $\begin{array}{l}r_{e} \\
(3)\end{array}$ & $\begin{array}{c}n \\
(4)\end{array}$ & $\begin{array}{l}\mu_{0} \\
(5)\end{array}$ & $\begin{array}{l}r_{0} \\
(6)\end{array}$ & $\begin{array}{l}M_{\text {Sersic }} \\
\quad(7)\end{array}$ & $\begin{array}{c}M_{\text {exp }} \\
\quad(8)\end{array}$ & $\begin{array}{c}M_{T} \\
(9)\end{array}$ & $\begin{array}{l}S / e \\
(10)\end{array}$ & $\begin{array}{c}\alpha \\
(11)\end{array}$ & $\begin{array}{l}e_{i n} \\
(12)\end{array}$ & $\begin{array}{c}e_{\text {out }} \\
(13)\end{array}$ & $\begin{array}{r}p a_{i n} \\
(14)\end{array}$ & $\begin{array}{c}p a_{o u t} \\
\quad(15)\end{array}$ & $\begin{array}{c}M_{\text {metric }} \\
\quad(16)\end{array}$ \\
\hline A 2415 & 23.75 & 31.01 & 6.94 & - & - & -24.20 & 0.00 & -24.20 & - & 0.60 & 0.21 & 0.35 & 27.1 & 25.5 & -22.76 \\
\hline A 2457 & 21.10 & 8.46 & 2.60 & 22.33 & 31.60 & -23.52 & -23.98 & -24.52 & 0.65 & 0.73 & 0.26 & 0.41 & 86.2 & 83.0 & -23.06 \\
\hline A 2459 & 21.25 & 8.03 & 2.36 & - & - & -23.25 & 0.00 & -23.25 & - & 0.49 & 0.28 & 0.26 & 13.2 & 3.0 & -22.68 \\
\hline A 2462 & 20.29 & 4.35 & 5.13 & 22.37 & 26.20 & -23.40 & -23.70 & -24.32 & 0.76 & 0.61 & 0.03 & 0.26 & -89.8 & -52.5 & -23.05 \\
\hline A 2480 & 19.86 & 3.45 & 3.82 & 22.23 & 22.80 & -23.16 & -23.51 & -24.10 & 0.72 & 0.51 & 0.03 & 0.48 & -18.0 & -40.0 & -23.00 \\
\hline A 2492 & 20.93 & 5.61 & 2.81 & 22.25 & 18.50 & -22.98 & -23.04 & -23.76 & 0.95 & 0.63 & 0.13 & 0.29 & -70.0 & 89.6 & -22.80 \\
\hline A 2495 & 21.70 & 6.56 & 1.30 & 21.52 & 26.60 & -22.20 & -24.59 & -24.71 & 0.11 & 1.01 & 0.18 & 0.43 & 50.4 & 50.7 & -22.70 \\
\hline A 2511 & 21.60 & 7.66 & 2.83 & - & - & -22.92 & 0.00 & -22.92 & - & 0.49 & 0.22 & 0.22 & 81.3 & 75.1 & -22.31 \\
\hline A 2524 & 21.19 & 7.10 & 4.65 & 22.03 & 17.90 & -23.44 & -23.14 & -24.05 & 1.33 & 0.63 & 0.19 & 0.28 & 14.5 & 1.9 & -22.93 \\
\hline A 2525 & 20.52 & 3.78 & 4.31 & 23.00 & 22.60 & -22.69 & -22.66 & -23.43 & 1.03 & 0.56 & 0.07 & 0.16 & -61.4 & -48.7 & -22.35 \\
\hline A2559 & 23.97 & 36.62 & 5.18 & 21.65 & 22.80 & -24.28 & -24.04 & -24.91 & 1.25 & 0.86 & 0.29 & 0.53 & 29.7 & 31.8 & -23.01 \\
\hline A 2572 & 19.18 & 2.54 & 1.37 & 21.31 & 17.30 & -22.43 & -23.62 & -23.93 & 0.34 & 0.50 & 0.23 & 0.18 & -85.8 & -19.2 & -22.81 \\
\hline A 2589 & 28.54 & 725.91 & 9.52 & - & - & -26.43 & 0.00 & -26.43 & - & 0.78 & 0.12 & 0.51 & -11.1 & 5.0 & -22.96 \\
\hline A 2593 & 21.93 & 6.60 & 4.83 & 20.54 & 12.00 & -22.40 & -23.65 & -23.95 & 0.33 & 0.90 & 0.18 & 0.38 & 75.2 & 73.8 & -22.44 \\
\hline A 2596 & 19.91 & 2.30 & 1.02 & 20.50 & 6.10 & -21.62 & -22.44 & -22.86 & 0.47 & 0.55 & 0.05 & 0.16 & -76.0 & -70.8 & -22.47 \\
\hline A 2618 & 20.79 & 6.60 & 1.69 & 21.94 & 25.50 & -23.11 & -23.95 & -24.36 & 0.46 & 0.80 & 0.06 & 0.26 & 77.2 & -86.4 & -23.15 \\
\hline A 2622 & 24.53 & 51.76 & 6.13 & - & - & -24.49 & 0.00 & -24.49 & - & 0.78 & 0.20 & 0.42 & -43.3 & -55.4 & -22.78 \\
\hline A 2625 & 21.60 & 10.49 & 3.10 & - & - & -23.67 & 0.00 & -23.67 & - & 0.54 & 0.37 & 0.36 & 84.9 & 85.9 & -22.78 \\
\hline A 2626 & 24.10 & 37.52 & 4.48 & 21.48 & 17.00 & -24.14 & -23.58 & -24.65 & 1.67 & 1.05 & 0.24 & 0.37 & 32.3 & 32.0 & -22.92 \\
\hline A 2630 & 22.56 & 15.00 & 5.56 & - & - & -23.83 & 0.00 & -23.83 & - & 0.59 & 0.05 & 0.31 & -49.4 & -50.5 & -22.87 \\
\hline A2634 & 20.33 & 4.66 & 3.16 & 21.27 & 20.60 & -22.98 & -24.00 & -24.35 & 0.39 & 0.67 & 0.18 & 0.45 & 31.0 & 34.3 & -22.98 \\
\hline A 2637 & 23.01 & 22.64 & 6.58 & - & - & -24.38 & 0.00 & -24.38 & - & 0.58 & 0.21 & 0.37 & 62.9 & 86.1 & -23.17 \\
\hline A2644 & 23.31 & 22.24 & 5.46 & - & - & -23.94 & 0.00 & -23.94 & - & 0.57 & 0.19 & 0.30 & 16.9 & 28.3 & -22.73 \\
\hline A2656 & 20.46 & 3.20 & 4.29 & 21.22 & 13.20 & -22.48 & -23.37 & -23.76 & 0.44 & 0.81 & 0.09 & 0.22 & -27.1 & -8.4 & -22.81 \\
\hline A 2657 & 21.24 & 2.74 & 3.94 & 19.76 & 3.50 & -21.04 & -21.67 & -22.15 & 0.56 & 0.36 & 0.11 & 0.07 & 1.8 & -9.3 & -21.95 \\
\hline A 2660 & 22.08 & 10.53 & 5.62 & 22.42 & 20.60 & -23.40 & -22.94 & -23.95 & 1.53 & 0.63 & 0.25 & 0.45 & -35.3 & -27.8 & -22.65 \\
\hline A2661 & 26.51 & 104.60 & 6.13 & - & - & -24.53 & 0.00 & -24.53 & - & 0.92 & 0.28 & 0.30 & -76.0 & -36.4 & -22.27 \\
\hline A2665 & 23.94 & 50.12 & 4.15 & - & - & -24.77 & 0.00 & -24.77 & - & 0.93 & 0.13 & 0.32 & -64.7 & -80.7 & -23.00 \\
\hline A2666 & 22.97 & 25.07 & 6.37 & - & - & -24.34 & 0.00 & -24.34 & - & 0.61 & 0.26 & 0.32 & 60.6 & 60.7 & -23.05 \\
\hline A 2670 & 21.13 & 7.49 & 5.43 & 21.75 & 22.30 & -23.69 & -23.88 & -24.54 & 0.84 & 0.70 & 0.09 & 0.08 & 81.4 & -39.7 & -23.28 \\
\hline A 2675 & 24.31 & 40.53 & 6.45 & - & - & -24.24 & 0.00 & -24.24 & - & 0.71 & 0.09 & 0.44 & 43.8 & 45.0 & -22.78 \\
\hline A 2678 & 21.85 & 8.54 & 6.41 & - & - & -23.32 & 0.00 & -23.32 & - & 0.48 & 0.18 & 0.23 & 17.7 & 19.0 & -22.55 \\
\hline A 2716 & 20.71 & 5.35 & 6.45 & - & - & -23.43 & 0.00 & -23.43 & - & 0.43 & 0.21 & 0.17 & -78.3 & -81.0 & -22.66 \\
\hline A 2717 & 20.15 & 2.80 & 2.84 & 21.25 & 18.60 & -22.08 & -23.88 & -24.07 & 0.19 & 0.87 & 0.05 & 0.18 & -17.3 & 8.3 & -22.64 \\
\hline A 2731 & 22.31 & 16.35 & 6.62 & - & - & -24.12 & 0.00 & -24.12 & - & 0.55 & 0.36 & 0.23 & 88.0 & 77.0 & -22.76 \\
\hline A 2734 & 21.70 & 9.87 & 1.27 & 22.08 & 32.30 & -22.91 & -24.29 & -24.56 & 0.28 & 1.03 & 0.19 & 0.50 & 21.5 & 21.6 & -22.76 \\
\hline A 2764 & 22.17 & 14.97 & 5.43 & - & - & -24.13 & 0.00 & -24.13 & - & 0.60 & 0.16 & 0.18 & -37.4 & 1.5 & -23.06 \\
\hline A 2765 & 19.33 & 2.05 & 4.59 & 21.75 & 9.30 & -22.58 & -21.98 & -23.08 & 1.74 & 0.54 & 0.24 & 0.29 & 51.7 & 51.8 & -22.09 \\
\hline A 2799 & 22.79 & 16.32 & 6.10 & - & - & -23.73 & 0.00 & -23.73 & - & 0.63 & 0.22 & 0.25 & 42.8 & 32.0 & -22.54 \\
\hline A 2800 & 22.38 & 14.39 & 4.42 & - & - & -23.70 & 0.00 & -23.70 & - & 0.62 & 0.25 & 0.42 & 63.4 & 75.7 & -22.71 \\
\hline A2806 & 19.85 & 3.12 & 3.32 & 21.11 & 7.20 & -22.59 & -21.86 & -23.04 & 1.97 & 0.46 & 0.16 & 0.20 & -62.5 & -58.7 & -22.60 \\
\hline A 2814 & 22.20 & 12.53 & 5.10 & - & - & -23.73 & 0.00 & -23.73 & - & 0.59 & 0.21 & 0.26 & -34.6 & -31.0 & -22.78 \\
\hline A 2819 & 20.27 & 5.19 & 2.62 & - & - & -23.36 & 0.00 & -23.36 & - & 0.63 & 0.24 & 0.18 & 42.4 & 35.2 & -22.62 \\
\hline A 2824 & 21.68 & 7.93 & 5.26 & - & - & -23.29 & 0.00 & -23.29 & - & 0.45 & 0.14 & 0.03 & 65.4 & 30.1 & -22.51 \\
\hline A 2836 & 21.80 & 6.84 & 2.75 & - & - & -22.46 & 0.00 & -22.46 & - & 0.49 & 0.07 & 0.10 & -53.4 & -37.0 & -22.12 \\
\hline A 2841 & 21.11 & 7.02 & 4.93 & 22.16 & 20.23 & -23.46 & -23.21 & -24.10 & 1.26 & 0.63 & 0.26 & 0.36 & -14.2 & -10.8 & -22.87 \\
\hline A 2854 & 21.39 & 4.80 & 5.59 & 22.11 & 24.50 & -22.41 & -23.66 & -23.96 & 0.32 & 0.65 & 0.18 & 0.50 & -2.6 & -15.7 & -22.28 \\
\hline A 2859 & 20.74 & 4.48 & 5.29 & 22.48 & 15.90 & -22.91 & -22.37 & -23.43 & 1.64 & 0.53 & 0.15 & 0.23 & -89.4 & -77.9 & -22.92 \\
\hline A 2864 & 18.86 & 1.55 & 5.08 & 21.75 & 10.20 & -22.48 & -22.16 & -23.08 & 1.34 & 0.55 & 0.17 & 0.26 & 28.6 & 34.4 & -22.17 \\
\hline A 2870 & 22.96 & 22.83 & 4.63 & - & - & -23.97 & 0.00 & -23.97 & - & 0.70 & 0.27 & 0.24 & 10.5 & 11.6 & -22.72 \\
\hline A 2877 & 19.91 & 5.63 & 3.68 & 21.04 & 19.90 & -23.63 & -23.89 & -24.52 & 0.78 & 0.68 & 0.14 & 0.28 & -69.5 & -92.9 & -23.44 \\
\hline A 2881 & 19.41 & 2.00 & 2.67 & 21.66 & 8.70 & -22.12 & -21.88 & -22.76 & 1.26 & 0.46 & 0.16 & 0.15 & 46.4 & 65.0 & -22.22 \\
\hline A 2889 & 19.88 & 1.44 & 1.01 & 22.05 & 5.70 & -20.29 & -20.41 & -21.10 & 0.90 & 0.34 & 0.42 & 0.38 & -44.4 & -41.4 & -20.49 \\
\hline A2896 & 18.95 & 2.21 & 3.68 & 21.01 & 8.50 & -22.71 & -22.23 & -23.25 & 1.56 & 0.40 & 0.27 & 0.33 & -62.6 & -65.8 & -22.44 \\
\hline A2911 & 19.64 & 2.34 & 2.82 & 22.01 & 11.90 & -21.72 & -21.67 & -22.45 & 1.04 & 0.47 & 0.17 & 0.23 & -18.4 & 14.5 & -21.78 \\
\hline A2923 & 22.24 & 13.42 & 4.15 & - & - & $\begin{array}{l}-23.69 \\
\end{array}$ & 0.00 & -23.69 & - & 0.66 & 0.03 & 0.29 & 66.1 & 75.8 & -22.89 \\
\hline A 2954 & 20.76 & 3.80 & 1.68 & - & - & -22.04 & 0.00 & -22.04 & - & 0.32 & 0.18 & 0.26 & 27.3 & 33.6 & -21.73 \\
\hline A2955 & 19.71 & 2.58 & $\begin{array}{l}1.00 \\
5.21\end{array}$ & 21.53 & 19.00 & -22.92 & -23.89 & $\begin{array}{l}-24.27 \\
-24\end{array}$ & 0.41 & 0.74 & 0.11 & 0.49 & 78.6 & 67.6 & -22.84 \\
\hline
\end{tabular}


TABLE 2-Continued

\begin{tabular}{|c|c|c|c|c|c|c|c|c|c|c|c|c|c|c|c|}
\hline $\begin{array}{l}\text { Name } \\
(1)\end{array}$ & $\begin{array}{l}\mu_{e} \\
(2)\end{array}$ & $\begin{array}{l}r_{e} \\
(3)\end{array}$ & $\begin{array}{c}n \\
(4)\end{array}$ & $\begin{array}{l}\mu_{0} \\
(5)\end{array}$ & $\begin{array}{l}r_{0} \\
(6)\end{array}$ & $\begin{array}{l}M_{\text {Sersic }} \\
\quad(7)\end{array}$ & $\begin{array}{c}M_{\text {exp }} \\
\quad(8)\end{array}$ & $\begin{array}{c}M_{T} \\
(9)\end{array}$ & $\begin{array}{l}S / e \\
(10)\end{array}$ & $\begin{array}{c}\alpha \\
(11)\end{array}$ & $\begin{array}{l}e_{i n} \\
(12)\end{array}$ & $\begin{array}{c}e_{\text {out }} \\
(13)\end{array}$ & $\begin{array}{r}p a_{i n} \\
(14)\end{array}$ & $\begin{array}{c}p a_{o u t} \\
\quad(15)\end{array}$ & $\begin{array}{c}M_{\text {metric }} \\
\quad(16)\end{array}$ \\
\hline A 2992 & 21.38 & 7.57 & 4.69 & - & - & -23.31 & 0.00 & -23.31 & - & 0.43 & 0.29 & 0.30 & -59.6 & -53.9 & -22.51 \\
\hline A3004 & 22.51 & 13.82 & 5.24 & - & - & -23.58 & 0.00 & -23.58 & - & 0.63 & 0.12 & 0.13 & -54.9 & 37.2 & -22.59 \\
\hline A3009 & 23.29 & 32.22 & 3.58 & - & - & -24.43 & 0.00 & -24.43 & - & 0.88 & 0.16 & 0.44 & -71.3 & -74.5 & -23.02 \\
\hline A3027 & 23.36 & 20.33 & 5.75 & 23.01 & 32.60 & -23.67 & -23.46 & -24.32 & 1.22 & 0.69 & 0.26 & 0.38 & 84.0 & 85.7 & -22.62 \\
\hline A 3047 & 21.99 & 8.22 & 4.55 & 21.93 & 23.03 & -23.01 & -23.84 & -24.26 & 0.46 & 0.81 & 0.22 & 0.45 & -59.4 & -48.3 & -22.80 \\
\hline A 3074 & 23.73 & 45.61 & 4.33 & - & - & -24.88 & 0.00 & -24.88 & - & 0.84 & 0.32 & 0.43 & 35.2 & 33.8 & -23.04 \\
\hline A3077 & 22.53 & 11.53 & 4.55 & - & - & -23.17 & 0.00 & -23.17 & - & 0.50 & 0.46 & 0.49 & 74.3 & 67.3 & -22.08 \\
\hline A3078 & 23.86 & 38.66 & 4.83 & - & - & -24.45 & 0.00 & -24.45 & - & 0.81 & 0.16 & 0.32 & 30.6 & 16.0 & -22.90 \\
\hline A3089 & 20.44 & 3.42 & 4.65 & 21.50 & 10.40 & -22.55 & -22.43 & -23.24 & 1.12 & 0.61 & 0.17 & 0.29 & 76.4 & 79.6 & -22.41 \\
\hline A3093 & 22.00 & 10.57 & 3.44 & 21.64 & 18.40 & -23.34 & -23.60 & -24.23 & 0.79 & 0.81 & 0.28 & 0.46 & 20.3 & 18.8 & -22.89 \\
\hline A3094 & 22.38 & 15.17 & 5.24 & - & - & -23.92 & 0.00 & -23.92 & - & 0.69 & 0.16 & 0.17 & 34.3 & 52.3 & -22.84 \\
\hline A3095 & 19.63 & 2.88 & 3.11 & 21.50 & 11.20 & -22.77 & -22.59 & -23.43 & 1.18 & 0.48 & 0.15 & 0.31 & -5.3 & -16.0 & -22.75 \\
\hline A3098 & 26.40 & 186.16 & 6.90 & - & - & -25.55 & 0.00 & -25.55 & - & 0.84 & 0.24 & 0.48 & -40.0 & -39.4 & -22.80 \\
\hline A3100 & 21.92 & 10.00 & 2.11 & - & - & -22.97 & 0.00 & -22.97 & - & 0.73 & 0.32 & 0.14 & 67.0 & 70.4 & -22.33 \\
\hline A3104 & 18.96 & 2.03 & 3.22 & 21.38 & 21.04 & -22.73 & -24.11 & -24.38 & 0.28 & 0.66 & 0.25 & 0.45 & 63.0 & 68.7 & -22.77 \\
\hline A3107 & 20.72 & 4.07 & 3.28 & 22.92 & 25.49 & -22.47 & -22.96 & -23.50 & 0.63 & 0.54 & 0.19 & 0.18 & -30.2 & -44.7 & -22.21 \\
\hline A3108 & 22.79 & 12.73 & 4.67 & - & - & -23.04 & 0.00 & -23.04 & - & 0.58 & 0.25 & 0.36 & 70.5 & 65.7 & -22.08 \\
\hline A3109 & 24.03 & 47.17 & 6.41 & - & - & -24.81 & 0.00 & -24.81 & - & 0.71 & 0.33 & 0.16 & 16.0 & 22.2 & -22.89 \\
\hline A3110 & 20.30 & 6.54 & 1.89 & 20.87 & 12.80 & -23.67 & -23.55 & -24.36 & 1.11 & 0.61 & 0.50 & 0.39 & -2.1 & -2.1 & -23.21 \\
\hline A3111 & 21.72 & 7.84 & 5.18 & 21.70 & 16.70 & -23.18 & -23.31 & -24.00 & 0.89 & 0.75 & 0.12 & 0.14 & 33.2 & 62.9 & -22.87 \\
\hline A 3112 & 24.95 & 71.55 & 6.54 & 22.01 & 33.50 & -24.87 & -24.50 & -25.45 & 1.40 & 0.88 & 0.33 & 0.59 & 9.2 & 6.7 & -23.06 \\
\hline A 3120 & 21.67 & 8.16 & 4.18 & 22.01 & 24.50 & -23.03 & -23.67 & -24.15 & 0.56 & 0.79 & 0.27 & 0.48 & -26.5 & -37.1 & -22.57 \\
\hline A 3122 & 20.51 & 5.83 & 5.78 & 22.82 & 27.10 & -23.76 & -23.20 & -24.27 & 1.68 & 0.60 & 0.27 & 0.39 & 38.0 & 61.4 & -22.96 \\
\hline A 3123 & 18.69 & 1.70 & 3.60 & 20.69 & 8.10 & -22.63 & -22.68 & -23.41 & 0.95 & 0.54 & 0.33 & 0.34 & -81.9 & -64.7 & -22.65 \\
\hline A 3125 & 19.83 & 2.86 & 3.04 & 21.83 & 9.70 & -22.52 & -21.92 & -23.01 & 1.73 & 0.49 & 0.07 & 0.13 & -2.9 & -34.0 & -22.46 \\
\hline A 3128 & 23.58 & 32.96 & 6.10 & - & - & -24.45 & 0.00 & -24.45 & - & 0.67 & 0.19 & 0.20 & 11.3 & 25.5 & -22.99 \\
\hline A3133 & 24.37 & 25.14 & 6.80 & - & - & -23.18 & 0.00 & -23.18 & - & 0.59 & 0.25 & 0.36 & -26.8 & -29.0 & -21.82 \\
\hline A3135 & 22.71 & 14.77 & 5.88 & - & - & -23.56 & 0.00 & -23.56 & - & 0.56 & 0.13 & 0.27 & 33.2 & 51.3 & -22.57 \\
\hline A 3142 & 20.80 & 5.23 & 3.32 & 21.92 & 17.64 & -22.94 & -23.16 & -23.81 & 0.81 & 0.64 & 0.21 & 0.30 & -12.1 & -21.4 & -22.71 \\
\hline A 3144 & 22.52 & 11.20 & 7.35 & - & - & -23.20 & 0.00 & -23.20 & - & 0.48 & 0.14 & 0.12 & -69.1 & -26.7 & -22.38 \\
\hline A3151 & 18.38 & 1.22 & 2.70 & 21.27 & 11.10 & -22.09 & -22.81 & -23.27 & 0.52 & 0.70 & 0.09 & 0.28 & -67.3 & -17.0 & -22.33 \\
\hline A3152 & 23.10 & 20.44 & 6.29 & - & - & -24.05 & 0.00 & -24.05 & - & 0.56 & 0.32 & 0.34 & 0.9 & 2.6 & -22.59 \\
\hline A3153 & 23.84 & 25.55 & 5.78 & - & - & -23.85 & 0.00 & -23.85 & - & 0.71 & 0.16 & 0.39 & 87.1 & 92.6 & -22.56 \\
\hline A3158 & 23.45 & 30.76 & 6.02 & - & - & -24.43 & 0.00 & -24.43 & - & 0.73 & 0.11 & 0.14 & 63.3 & 59.7 & -23.06 \\
\hline A3164 & 23.18 & 20.63 & 7.04 & - & - & -23.91 & 0.00 & -23.91 & - & 0.59 & 0.10 & 0.20 & -59.8 & 30.3 & -22.94 \\
\hline A3188 & 19.77 & 2.00 & 6.02 & - & - & -22.19 & 0.00 & -22.19 & - & 0.32 & 0.20 & 0.28 & -50.7 & -66.8 & -21.40 \\
\hline A3193 & 23.10 & 19.45 & 6.49 & - & - & -23.72 & 0.00 & -23.72 & - & 0.54 & 0.10 & 0.24 & -82.5 & 87.3 & -22.57 \\
\hline A3195 & 23.28 & 22.96 & 5.99 & - & - & -24.02 & 0.00 & -24.02 & - & 0.67 & 0.32 & 0.39 & 64.0 & 60.6 & -22.72 \\
\hline A 3223 & 20.01 & 4.49 & 1.84 & 21.61 & 16.00 & -23.06 & -23.23 & -23.90 & 0.85 & 0.56 & 0.26 & 0.26 & 0.6 & -6.8 & -22.98 \\
\hline A3225 & 19.61 & 2.40 & 0.93 & 20.28 & 7.50 & -21.74 & -22.88 & -23.21 & 0.35 & 0.64 & 0.13 & 0.16 & -11.3 & -8.0 & -22.68 \\
\hline A3229 & 20.06 & 2.40 & 2.07 & - & - & -21.54 & 0.00 & -21.54 & - & 0.24 & 0.46 & 0.48 & -78.7 & -85.9 & -21.07 \\
\hline A 3231 & 22.28 & 7.98 & 5.56 & - & - & -22.69 & 0.00 & -22.69 & - & 0.49 & 0.07 & 0.15 & 84.4 & 84.5 & -21.90 \\
\hline A3234 & 21.60 & 7.72 & 4.93 & - & - & -23.41 & 0.00 & -23.41 & - & 0.49 & 0.20 & 0.13 & 27.9 & 33.3 & -22.55 \\
\hline A3266 & 20.23 & 4.04 & 5.59 & 21.02 & 26.10 & -23.20 & -24.89 & -25.09 & 0.21 & 0.83 & 0.12 & 0.31 & 77.4 & 63.4 & -23.19 \\
\hline A3301 & 21.83 & 13.08 & 3.11 & 23.26 & 80.22 & -23.81 & -25.06 & -25.36 & 0.32 & 0.81 & 0.07 & 0.37 & -45.8 & -42.7 & -23.15 \\
\hline A3332 & 20.94 & 5.94 & 4.12 & 23.16 & 36.36 & -23.24 & -23.55 & -24.16 & 0.75 & 0.52 & 0.12 & 0.44 & 48.5 & 47.7 & -22.83 \\
\hline A3336 & 22.95 & 20.24 & 4.12 & 23.28 & 42.50 & -23.90 & -23.77 & -24.59 & 1.12 & 0.82 & 0.22 & 0.34 & 19.5 & 17.8 & -22.82 \\
\hline A3341 & 21.09 & 4.95 & 6.06 & 21.71 & 16.80 & -22.72 & -23.14 & -23.70 & 0.68 & 0.72 & 0.21 & 0.33 & 11.8 & 21.7 & -22.44 \\
\hline A3351 & 21.63 & 7.82 & 6.25 & - & - & -23.43 & 0.00 & -23.43 & - & 0.52 & 0.10 & 0.06 & -34.1 & 71.5 & -22.58 \\
\hline A3354 & 20.15 & 3.80 & 2.38 & 22.75 & 22.40 & -22.69 & -22.82 & -23.51 & 0.89 & 0.47 & 0.09 & 0.15 & 3.9 & 1.2 & -22.63 \\
\hline A3367 & 21.91 & 10.08 & 3.46 & - & - & -23.19 & 0.00 & -23.19 & - & 0.39 & 0.27 & 0.35 & 52.1 & 54.9 & -22.24 \\
\hline A3374 & 14.82 & 0.24 & 2.79 & 19.08 & 4.00 & -22.02 & -22.67 & -23.14 & 0.55 & 0.46 & 0.44 & 0.40 & -70.5 & -70.6 & -22.17 \\
\hline A3376 & 20.91 & 7.24 & 2.87 & 22.28 & 30.50 & -23.37 & -23.91 & -24.43 & 0.61 & 0.68 & 0.28 & 0.44 & 64.4 & 67.9 & -22.87 \\
\hline A3380 & 21.71 & 7.91 & 5.38 & 23.40 & 56.80 & -23.12 & -24.16 & -24.51 & 0.38 & 0.58 & 0.07 & 0.41 & 81.2 & 69.2 & -22.56 \\
\hline A3381 & 24.08 & 29.06 & 6.45 & - & - & -23.60 & 0.00 & -23.60 & - & 0.64 & 0.11 & 0.18 & 36.0 & 29.9 & -22.28 \\
\hline A3389 & 21.84 & 13.47 & 3.07 & - & - & -23.77 & 0.00 & -23.77 & - & 0.69 & 0.18 & 0.16 & 60.3 & 19.8 & -22.88 \\
\hline A3390 & 21.20 & 5.15 & 5.65 & 21.91 & 15.90 & -22.66 & -22.83 & -23.50 & 0.86 & 0.65 & 0.04 & 0.12 & -79.5 & -75.0 & -22.55 \\
\hline A3391 & 22.44 & 22.42 & 3.31 & - & 10.80 & -24.41 & 0.00 & -24.41 & 0.00 & 0.74 & $\begin{array}{l}0.04 \\
0.24\end{array}$ & 0.38 & 76.1 & 45.6 & -23.15 \\
\hline
\end{tabular}


TABLE 2-Continued

\begin{tabular}{|c|c|c|c|c|c|c|c|c|c|c|c|c|c|c|c|}
\hline $\begin{array}{l}\text { Name } \\
(1)\end{array}$ & $\begin{array}{l}\mu_{e} \\
(2)\end{array}$ & $\begin{array}{l}r_{e} \\
(3)\end{array}$ & $\begin{array}{c}n \\
(4)\end{array}$ & $\begin{array}{l}\mu_{0} \\
(5)\end{array}$ & $\begin{array}{l}r_{0} \\
(6)\end{array}$ & $\begin{array}{l}M_{\text {Sersic }} \\
\quad(7)\end{array}$ & $\begin{array}{c}M_{\text {exp }} \\
\quad(8)\end{array}$ & $\begin{array}{c}M_{T} \\
(9)\end{array}$ & $\begin{array}{l}S / e \\
(10)\end{array}$ & $\begin{array}{c}\alpha \\
(11)\end{array}$ & $\begin{array}{l}e_{i n} \\
(12)\end{array}$ & $\begin{array}{c}e_{\text {out }} \\
(13)\end{array}$ & $\begin{array}{l}p a_{i n} \\
(14)\end{array}$ & $\begin{array}{c}p a_{\text {out }} \\
(15)\end{array}$ & $\begin{array}{c}M_{\text {metric }} \\
(16)\end{array}$ \\
\hline A3395 & 26.19 & 202.47 & 5.78 & - & - & -25.71 & 0.00 & -25.71 & - & 0.88 & 0.38 & 0.57 & -56.2 & -63.4 & -22.56 \\
\hline A3404 & 24.18 & 56.46 & 3.28 & - & - & -25.09 & 0.00 & -25.09 & - & 1.14 & 0.29 & 0.39 & 66.6 & 70.6 & -22.94 \\
\hline A3407 & 25.64 & 99.66 & 7.87 & - & - & -24.90 & 0.00 & -24.90 & - & 0.74 & 0.08 & 0.02 & -88.9 & 34.5 & -22.84 \\
\hline A3408 & 22.46 & 16.24 & 5.78 & 23.25 & 46.90 & -23.93 & -23.85 & -24.64 & 1.07 & 0.64 & 0.08 & 0.33 & -43.7 & -39.8 & -23.02 \\
\hline A 3420 & 20.70 & 4.42 & 4.85 & 23.22 & 19.60 & -22.86 & -22.08 & -23.29 & 2.06 & 0.44 & 0.20 & 0.22 & 38.7 & 38.8 & -22.42 \\
\hline A 3429 & 24.66 & 53.10 & 7.04 & - & - & -24.43 & 0.00 & -24.43 & - & 0.70 & 0.21 & 0.47 & 39.7 & 38.8 & -22.67 \\
\hline A3490 & 21.28 & 6.22 & 4.81 & 22.69 & 26.70 & -23.17 & -23.43 & -24.06 & 0.79 & 0.67 & 0.13 & 0.26 & 37.5 & 57.1 & -22.73 \\
\hline A3497 & 24.02 & 32.71 & 5.46 & - & - & -23.97 & 0.00 & -23.97 & - & 0.75 & 0.18 & 0.29 & 50.6 & 43.8 & -22.47 \\
\hline A 3528 & 22.24 & 14.03 & 5.15 & 22.33 & 36.20 & -23.91 & -24.36 & -24.91 & 0.66 & 0.68 & 0.17 & 0.47 & 0.2 & -5.8 & -23.16 \\
\hline A3530 & 22.94 & 17.98 & 5.71 & 21.47 & 25.30 & -23.80 & -24.43 & -24.91 & 0.56 & 0.80 & 0.23 & 0.45 & -54.7 & -34.6 & -23.05 \\
\hline A3531 & 22.03 & 4.76 & 6.10 & 21.98 & 17.50 & -21.76 & -23.02 & -23.31 & 0.31 & 0.85 & 0.37 & 0.51 & 15.9 & 15.3 & -21.71 \\
\hline A3532 & 22.33 & 11.85 & 5.65 & 22.40 & 42.40 & -23.51 & -24.64 & -24.97 & 0.36 & 0.76 & 0.15 & 0.39 & 64.5 & 79.2 & -22.89 \\
\hline A3537 & 22.17 & 17.15 & 5.52 & - & - & -23.84 & 0.00 & -23.84 & - & 0.57 & 0.31 & 0.27 & -12.5 & -26.0 & -22.70 \\
\hline A3549 & 21.91 & 8.42 & 5.26 & - & - & -23.05 & 0.00 & -23.05 & - & 0.45 & 0.29 & 0.26 & 3.1 & 3.0 & -22.14 \\
\hline A3549 & 23.03 & 11.69 & 5.81 & - & - & -22.78 & 0.00 & -22.78 & - & 0.58 & 0.10 & 0.09 & -68.2 & 69.6 & -21.95 \\
\hline A3552 & 22.27 & 20.56 & 6.02 & - & - & -24.70 & 0.00 & -24.70 & - & 0.60 & 0.08 & 0.06 & 22.3 & 87.4 & -22.58 \\
\hline A3553 & 22.33 & 9.53 & 4.00 & - & - & -22.81 & 0.00 & -22.81 & - & 0.56 & 0.04 & 0.09 & -88.9 & 47.3 & -22.22 \\
\hline A3554 & 23.55 & 20.03 & 5.78 & 21.46 & 20.20 & -23.39 & -23.91 & -24.43 & 0.62 & 0.82 & 0.24 & 0.34 & -35.6 & -49.2 & -22.68 \\
\hline A3556 & 22.15 & 18.39 & 4.05 & - & - & -24.42 & 0.00 & -24.42 & - & 0.68 & 0.32 & 0.14 & -35.9 & -40.1 & -23.18 \\
\hline A3559 & 24.73 & 112.07 & 4.63 & - & - & -25.76 & 0.00 & -25.76 & - & 0.99 & 0.13 & 0.35 & -22.8 & -21.0 & -23.26 \\
\hline A3559 & 18.15 & 1.61 & 4.88 & 20.60 & 14.10 & -23.22 & -23.98 & -24.42 & 0.50 & 0.61 & 0.19 & 0.42 & 28.4 & 47.3 & -23.18 \\
\hline A3560 & 20.62 & 5.86 & 3.57 & - & - & -22.54 & 0.00 & -22.54 & - & 0.46 & 0.21 & 0.07 & 72.8 & -45.3 & -22.11 \\
\hline A3562 & 22.28 & 15.39 & 2.92 & 22.96 & 72.90 & -23.74 & -25.21 & -25.46 & 0.26 & 0.80 & 0.20 & 0.57 & 89.7 & 84.0 & -22.88 \\
\hline A3564 & 21.93 & 7.10 & 5.78 & - & - & -22.78 & 0.00 & -22.78 & - & 0.43 & 0.13 & 0.05 & -50.0 & -41.9 & -22.20 \\
\hline A3564 & 21.95 & 10.15 & 4.13 & - & - & -23.35 & 0.00 & -23.35 & - & 0.54 & 0.14 & 0.11 & -0.8 & -12.5 & -22.62 \\
\hline A3565 & 23.47 & 36.59 & 7.75 & - & - & -24.10 & 0.00 & -24.10 & - & 0.64 & 0.09 & 0.05 & 60.3 & 8.7 & -22.76 \\
\hline A 3570 & 20.62 & 4.88 & 4.22 & - & - & -22.99 & 0.00 & -22.99 & - & 0.39 & 0.15 & 0.12 & 5.2 & 73.2 & -22.50 \\
\hline A3571 & 25.51 & 232.87 & 4.57 & - & - & -26.54 & 0.00 & -26.54 & - & 1.16 & 0.24 & 0.64 & 1.7 & 3.8 & -23.14 \\
\hline A 3572 & 21.12 & 6.30 & 5.68 & - & - & -23.22 & 0.00 & -23.22 & - & 0.40 & 0.07 & 0.12 & 30.0 & -64.2 & -22.61 \\
\hline A3574 & 22.15 & 11.42 & 6.17 & 20.69 & 17.80 & -22.94 & -23.74 & -24.17 & 0.48 & 0.76 & 0.14 & 0.45 & 68.6 & 67.2 & -22.64 \\
\hline A3575 & 20.94 & 3.70 & 5.99 & - & - & -22.24 & 0.00 & -22.24 & - & 0.32 & 0.07 & 0.13 & -66.8 & -63.9 & -21.78 \\
\hline A3581 & 25.38 & 74.80 & 8.20 & - & - & -24.22 & 0.00 & -24.22 & - & 0.65 & 0.13 & 0.34 & -65.4 & -70.8 & -22.41 \\
\hline A3605 & 21.58 & 10.17 & 1.46 & 23.35 & 56.40 & -23.20 & -24.27 & -24.62 & 0.37 & 0.90 & 0.10 & 0.22 & 59.0 & 51.9 & -22.80 \\
\hline A3626 & 22.10 & 10.36 & 1.05 & - & - & -22.55 & 0.00 & -22.55 & - & 0.78 & 0.31 & 0.41 & 19.5 & 32.7 & -21.97 \\
\hline A3631 & 21.57 & 6.64 & 0.81 & - & - & -22.01 & 0.00 & -22.01 & - & 0.49 & 0.31 & 0.25 & -50.9 & -52.7 & -21.66 \\
\hline A3651 & 22.29 & 19.27 & 2.46 & - & - & -24.10 & 0.00 & -24.10 & - & 0.91 & 0.13 & 0.19 & -34.1 & -34.8 & -23.08 \\
\hline A3656 & 24.07 & 41.74 & 8.00 & - & - & -24.45 & 0.00 & -24.45 & - & 0.61 & 0.17 & 0.18 & -55.5 & -67.1 & -22.84 \\
\hline A3667 & 20.88 & 7.19 & 1.47 & 22.01 & 32.00 & -23.09 & -24.32 & -24.62 & 0.32 & 0.90 & 0.17 & 0.36 & 67.9 & -46.1 & -23.03 \\
\hline A3677 & 22.02 & 8.90 & 4.78 & - & - & -22.96 & 0.00 & -22.96 & - & 0.47 & 0.23 & 0.31 & -45.7 & -42.1 & -22.11 \\
\hline A3687 & 25.22 & 71.69 & 6.99 & - & - & -24.63 & 0.00 & -24.63 & - & 0.72 & 0.22 & 0.38 & -46.5 & -44.2 & -22.57 \\
\hline A3698 & 21.89 & 10.61 & 6.37 & - & - & -23.16 & 0.00 & -23.16 & - & 0.42 & 0.31 & 0.44 & 8.4 & 5.4 & -22.13 \\
\hline A 3703 & 23.23 & 19.73 & 6.13 & 23.09 & 41.10 & -23.73 & -23.84 & -24.54 & 0.90 & 0.70 & 0.22 & 0.47 & -87.8 & 79.9 & -22.59 \\
\hline A3716 & 22.34 & 11.69 & 5.88 & 21.57 & 20.00 & -23.39 & -23.74 & -24.33 & 0.73 & 0.78 & 0.05 & 0.32 & 63.5 & 56.7 & -22.91 \\
\hline A3731 & 20.21 & 4.32 & 3.04 & 22.32 & 23.17 & -22.99 & -23.28 & -23.90 & 0.77 & 0.56 & 0.11 & 0.30 & -13.0 & -0.3 & -22.80 \\
\hline A3733 & 20.21 & 2.79 & 1.22 & 21.23 & 11.40 & -21.47 & -22.71 & -23.01 & 0.32 & 0.64 & 0.13 & 0.20 & 7.3 & -2.2 & -22.16 \\
\hline A3736 & 24.14 & 65.96 & 5.85 & - & - & -25.33 & 0.00 & -25.33 & - & 0.79 & 0.31 & 0.48 & 24.4 & 23.9 & -23.24 \\
\hline A 3742 & 21.57 & 8.81 & 7.04 & - & - & -23.01 & 0.00 & -23.01 & - & 0.45 & 0.28 & 0.14 & -46.5 & -39.1 & -22.15 \\
\hline A 3744 & 22.33 & 12.68 & 6.37 & - & - & -23.48 & 0.00 & -23.48 & - & 0.55 & 0.02 & 0.11 & 39.8 & 12.8 & -22.65 \\
\hline A 3747 & 22.21 & 13.72 & 4.35 & - & - & -23.61 & 0.00 & -23.61 & - & 0.54 & 0.35 & 0.41 & 38.7 & 25.9 & -22.52 \\
\hline A 3756 & 22.03 & 10.94 & 5.18 & - & - & -23.49 & 0.00 & -23.49 & - & 0.50 & 0.22 & 0.31 & -37.2 & -43.4 & -22.60 \\
\hline A3764 & 22.36 & 12.13 & 6.25 & - & - & -23.60 & 0.00 & -23.60 & - & 0.56 & 0.12 & 0.23 & -68.2 & -31.7 & -22.70 \\
\hline A3771 & 19.25 & 2.00 & 2.44 & 21.84 & 14.90 & -22.28 & -22.92 & -23.40 & 0.56 & 0.58 & 0.16 & 0.26 & 21.6 & 10.3 & -22.44 \\
\hline A 3775 & 18.69 & 1.56 & 2.56 & 20.44 & 8.30 & -22.44 & -23.16 & -23.61 & 0.51 & 0.61 & 0.20 & 0.04 & -4.3 & 84.7 & -22.63 \\
\hline A3781 & 22.30 & 7.13 & 6.10 & - & - & -22.40 & 0.00 & -22.40 & - & 0.56 & 0.05 & 0.24 & 72.8 & 39.8 & -21.74 \\
\hline A3782 & 22.70 & 16.45 & 5.56 & 23.07 & 36.40 & -23.75 & -23.54 & -24.41 & 1.22 & 0.70 & 0.08 & 0.37 & -20.2 & -52.9 & -22.89 \\
\hline A3785 & 22.46 & 16.31 & 5.62 & - & - & -24.07 & 0.00 & -24.07 & 1.22 & 0.61 & 0.15 & 0.14 & 5.2 & -13.3 & -23.05 \\
\hline A3796 & 21.98 & 8.89 & 5.26 & 22.47 & 23.20 & -23.19 & -23.24 & $\begin{array}{l}-23.97 \\
\end{array}$ & 0.95 & 0.65 & 0.22 & 0.32 & -27.8 & -43.8 & -22.60 \\
\hline A3806 & 21.05 & 8.54 & $\begin{array}{l}0.20 \\
1.44\end{array}$ & 22.26 & 31.00 & -23.36 & $\begin{array}{l}-24.08 \\
-24.08\end{array}$ & -24.53 & 0.52 & 0.90 & 0.14 & 0.21 & 67.4 & 62.2 & -23.12 \\
\hline
\end{tabular}


TABLE 2-Continued

\begin{tabular}{|c|c|c|c|c|c|c|c|c|c|c|c|c|c|c|c|}
\hline $\begin{array}{c}\text { Name } \\
(1)\end{array}$ & $\begin{array}{l}\mu_{e} \\
(2)\end{array}$ & $\begin{array}{l}r_{e} \\
(3)\end{array}$ & $\begin{array}{c}n \\
(4)\end{array}$ & $\begin{array}{l}\mu_{0} \\
(5)\end{array}$ & $\begin{array}{l}r_{0} \\
(6)\end{array}$ & $\begin{array}{c}M_{\text {Sersic }} \\
\quad(7)\end{array}$ & $\begin{array}{c}M_{\exp } \\
(8)\end{array}$ & $\begin{array}{r}M_{T} \\
(9)\end{array}$ & $\begin{array}{l}S / e \\
(10)\end{array}$ & $\begin{array}{c}\alpha \\
(11)\end{array}$ & $\begin{array}{l}e_{i n} \\
(12)\end{array}$ & $\begin{array}{l}e_{\text {out }} \\
(13)\end{array}$ & $\begin{array}{c}p a_{i n} \\
(14)\end{array}$ & $\begin{array}{c}p a_{\text {out }} \\
(15)\end{array}$ & $\begin{array}{c}M_{\text {metric }} \\
\quad(16)\end{array}$ \\
\hline A3809 & 26.22 & 144.88 & 7.30 & - & - & -25.16 & 0.00 & -25.16 & - & 0.81 & 0.13 & 0.44 & 87.0 & 88.9 & -22.70 \\
\hline A3822 & 24.77 & 66.64 & 6.41 & - & - & -24.87 & 0.00 & -24.87 & - & 0.68 & 0.30 & 0.37 & -22.8 & -19.0 & -22.87 \\
\hline A3825 & 17.26 & 0.61 & 4.05 & 20.68 & 4.40 & -21.79 & -21.26 & -22.31 & 1.62 & 0.28 & 0.34 & 0.26 & -46.8 & -67.6 & -21.53 \\
\hline A3826 & 22.59 & 26.06 & 3.19 & - & - & -24.65 & 0.00 & -24.65 & - & 0.81 & 0.35 & 0.38 & -22.6 & -23.0 & -23.17 \\
\hline A3836 & 22.31 & 15.43 & 4.98 & 22.58 & 64.40 & -24.16 & -25.49 & -25.77 & 0.29 & 0.80 & 0.15 & 0.43 & -45.5 & -23.7 & -23.30 \\
\hline A3837 & 23.70 & 21.38 & 6.67 & - & - & -23.56 & 0.00 & -23.56 & - & 0.58 & 0.14 & 0.16 & 7.2 & 0.7 & -22.45 \\
\hline A3844 & 20.25 & 2.77 & 4.37 & 24.30 & 56.02 & -22.28 & -23.32 & -23.67 & 0.38 & 0.41 & 0.13 & 0.21 & 57.3 & 107.8 & -21.75 \\
\hline A3851 & 20.01 & 3.52 & 1.16 & 20.95 & 11.90 & -22.29 & -23.23 & -23.61 & 0.42 & 0.56 & 0.28 & 0.27 & -88.6 & 78.1 & -22.68 \\
\hline A3864 & 26.28 & 226.18 & 6.67 & - & - & -26.15 & 0.00 & -26.15 & - & 0.88 & 0.25 & 0.58 & -45.9 & -44.6 & -23.18 \\
\hline A3869 & 21.94 & 10.51 & 6.41 & - & - & -23.50 & 0.00 & -23.50 & - & 0.45 & 0.19 & 0.27 & -51.1 & -52.5 & -22.61 \\
\hline A3879 & 19.19 & 2.12 & 3.89 & 21.61 & 11.50 & -22.67 & -22.55 & -23.36 & 1.13 & 0.56 & 0.23 & 0.25 & -52.4 & -37.5 & -22.42 \\
\hline A3880 & 23.46 & 31.92 & 3.83 & - & - & -24.24 & 0.00 & -24.24 & - & 0.89 & 0.05 & 0.38 & 20.9 & -37.4 & -22.85 \\
\hline A3886 & 22.04 & 11.71 & 5.05 & - & - & -23.69 & 0.00 & -23.69 & - & 0.54 & 0.20 & 0.15 & -2.7 & 5.1 & -22.74 \\
\hline A3895 & 23.38 & 25.51 & 3.91 & - & - & -23.85 & 0.00 & -23.85 & - & 0.75 & 0.23 & 0.36 & 2.5 & -6.2 & -22.61 \\
\hline A3912 & 21.36 & 7.55 & 0.99 & 21.97 & 16.90 & -22.57 & -23.02 & -23.57 & 0.66 & 0.91 & 0.17 & 0.26 & 34.8 & 15.8 & -22.62 \\
\hline A3915 & 22.41 & 12.82 & 6.45 & 23.36 & 29.33 & -23.74 & -22.95 & -24.17 & 2.08 & 0.71 & 0.17 & 0.29 & 86.9 & 87.3 & -22.85 \\
\hline A3959 & 17.17 & 0.62 & 3.18 & 20.67 & 6.00 & -21.92 & -22.07 & -22.75 & 0.87 & 0.46 & 0.42 & 0.45 & 22.6 & 20.3 & -21.72 \\
\hline A3969 & 21.92 & 11.74 & 4.78 & - & - & -24.00 & 0.00 & -24.00 & - & 0.67 & 0.13 & 0.08 & -24.5 & -106.5 & -23.02 \\
\hline A3985 & 23.46 & 38.82 & 3.07 & - & - & -24.74 & 0.00 & -24.74 & - & 0.92 & 0.44 & 0.61 & 30.5 & 30.4 & -22.55 \\
\hline A4008 & 22.76 & 21.08 & 4.29 & - & - & -24.10 & 0.00 & -24.10 & - & 0.71 & 0.22 & 0.34 & 57.3 & 51.8 & -22.91 \\
\hline A4038 & 23.76 & 31.17 & 7.41 & - & - & -23.96 & 0.00 & -23.96 & - & 0.57 & 0.15 & 0.36 & -39.3 & -47.1 & -22.60 \\
\hline A4049 & 21.75 & 9.04 & 5.32 & 23.39 & 43.40 & -23.26 & -23.48 & -24.12 & 0.81 & 0.57 & 0.08 & 0.12 & 66.6 & 47.8 & -22.71 \\
\hline A4053 & 21.81 & 9.74 & 7.35 & - & - & -23.63 & 0.00 & -23.63 & - & 0.51 & 0.10 & 0.12 & -85.0 & -71.0 & -22.71 \\
\hline A4059 & 24.17 & 77.05 & 4.41 & - & - & -25.49 & 0.00 & -25.49 & - & 0.98 & 0.22 & 0.41 & -22.1 & -23.2 & -23.21 \\
\hline
\end{tabular}

Note.-Col. (1), Abell Cluster; col (2), effective surface magnitude; col. (3), effective radius (kpc); col. (4), $n$ parameter; col. (5), central surface magnitude; col. (6), scale length (kpc); col. (7), Sersic absolute magnitude; col. (8), exponential absolute magnitude; col. (9), total absolute magnitude; col. (10), Sérsic/exponential ratio; col. (11), $\alpha$ parameter; col. (12), inner ellipticity; col. (13), outer ellipticity; col. (14), inner position angle ; col. (15), outer position angle; col. (16), Metric absolute magnitude. 
TABLE 3

BCGs Photometrical Parameters (Hopkins model)

\begin{tabular}{|c|c|c|c|c|c|c|c|c|c|}
\hline $\begin{array}{c}\text { Name } \\
(1)\end{array}$ & $\begin{array}{l}\mu_{e} \\
(2)\end{array}$ & $\begin{array}{l}r_{e} \\
(3)\end{array}$ & $\begin{array}{c}n \\
(4)\end{array}$ & $\begin{array}{l}\mu_{0} \\
(5)\end{array}$ & $\begin{array}{l}r_{0} \\
(6)\end{array}$ & $\begin{array}{c}M_{\text {Sersic }} \\
\quad(7)\end{array}$ & $\begin{array}{c}M_{\text {exp }} \\
\quad(8)\end{array}$ & $\begin{array}{c}M_{T} \\
(9)\end{array}$ & $\begin{array}{l}e / S \\
(10)\end{array}$ \\
\hline A0085 & 23.72 & 47.0 & 0.6 & 21.42 & 14.3 & -23.91 & -23.84 & -24.63 & 0.935 \\
\hline A0133 & 23.23 & 39.0 & 1.2 & 21.04 & 6.9 & -24.29 & -22.66 & -24.51 & 0.222 \\
\hline A0150 & 24.34 & 49.2 & 3.5 & 20.84 & 3.3 & -24.22 & -21.25 & -24.29 & 0.065 \\
\hline A0152 & 22.77 & 20.5 & 1.4 & 19.63 & 1.7 & -23.54 & -21.07 & -23.65 & 0.102 \\
\hline A0193 & 25.18 & 106.4 & 3.5 & 18.99 & 1.9 & -25.04 & -21.86 & -25.10 & 0.053 \\
\hline A0194 & 22.05 & 14.2 & 1.7 & 18.39 & 1.1 & -23.31 & -21.09 & -23.44 & 0.130 \\
\hline A0208 & 25.42 & 92.5 & 4.6 & 20.24 & 2.5 & -24.87 & -21.43 & -24.91 & 0.042 \\
\hline $\mathrm{A} 0257$ & 22.64 & 18.5 & 1.9 & 19.72 & 2.1 & -23.68 & -21.58 & -23.82 & 0.144 \\
\hline A0260 & 24.83 & 69.3 & 5.7 & 19.43 & 1.6 & -24.66 & -21.04 & -24.70 & 0.036 \\
\hline A0262 & 23.41 & 34.9 & 1.8 & 20.07 & 2.4 & -23.69 & -20.92 & -23.77 & 0.078 \\
\hline A0268 & 23.80 & 17.9 & 4.0 & 20.18 & 1.9 & -22.77 & -20.87 & -22.95 & 0.173 \\
\hline A 0279 & 24.02 & 52.5 & 3.1 & 20.46 & 3.3 & -24.73 & -21.70 & -24.79 & 0.061 \\
\hline A 0295 & 23.15 & 25.6 & 3.5 & 19.36 & 1.4 & -23.95 & -20.76 & -24.01 & 0.053 \\
\hline A0311 & 25.52 & 146.2 & 3.6 & 20.73 & 3.6 & -25.48 & -21.57 & -25.51 & 0.027 \\
\hline A0386 & 22.21 & 8.8 & 2.3 & 19.40 & 1.8 & -22.54 & -21.47 & -22.88 & 0.375 \\
\hline A0397 & 23.88 & 37.9 & 2.9 & 19.39 & 2.0 & -23.98 & -21.50 & -24.09 & 0.101 \\
\hline A0399 & 23.88 & 62.9 & 1.8 & 21.90 & 7.6 & -24.97 & -22.07 & -25.05 & 0.069 \\
\hline A0404 & 23.08 & 21.0 & 1.5 & 20.35 & 3.7 & -23.26 & -22.01 & -23.56 & 0.316 \\
\hline A0415 & 23.51 & 40.2 & 1.4 & 20.99 & 4.2 & -24.88 & -22.32 & -24.98 & 0.095 \\
\hline A0498 & 25.12 & 73.3 & 3.8 & 20.83 & 2.6 & -24.48 & -20.85 & -24.52 & 0.035 \\
\hline A0500 & 25.48 & 97.6 & 4.8 & 20.32 & 3.1 & -24.91 & -21.82 & -24.97 & 0.058 \\
\hline A0539 & 24.12 & 39.2 & 3.8 & 19.37 & 1.7 & -23.94 & -21.24 & -24.03 & 0.083 \\
\hline A0548 & 21.35 & 10.1 & 1.6 & 19.62 & 1.6 & -23.32 & -20.84 & -23.42 & 0.102 \\
\hline A0553 & 24.88 & 65.0 & 4.5 & 20.60 & 3.2 & -24.58 & -21.57 & -24.65 & 0.062 \\
\hline A0564 & 28.01 & 334.8 & 4.3 & 20.12 & 3.7 & -25.03 & -22.43 & -25.13 & 0.091 \\
\hline A0564 & 27.66 & 267.4 & 4.0 & 20.12 & 3.7 & -24.86 & -22.43 & -24.97 & 0.107 \\
\hline A0582 & 24.16 & 29.6 & 3.6 & 19.78 & 2.1 & -23.45 & -21.42 & -23.61 & 0.154 \\
\hline A0671 & 23.68 & 51.2 & 2.9 & 19.72 & 3.1 & -24.87 & -22.19 & -24.96 & 0.085 \\
\hline A0690 & 24.86 & 69.3 & 4.6 & 20.24 & 2.9 & -24.74 & -21.71 & -24.80 & 0.062 \\
\hline A 0757 & 23.71 & 19.1 & 3.4 & 20.67 & 2.9 & -22.80 & -21.10 & -23.00 & 0.210 \\
\hline A0779 & 23.86 & 59.1 & 4.9 & 19.21 & 2.0 & -24.89 & -21.35 & -24.93 & 0.038 \\
\hline A 0780 & 23.55 & 37.2 & 3.1 & 19.28 & 1.6 & -24.36 & -21.22 & -24.42 & 0.055 \\
\hline A0834 & 21.45 & 7.6 & 0.8 & 19.92 & 2.0 & -22.39 & -21.08 & -22.68 & 0.299 \\
\hline A0841 & 24.10 & 44.4 & 3.3 & 19.95 & 2.8 & -24.29 & -21.86 & -24.40 & 0.106 \\
\hline A0957 & 23.22 & 60.2 & 2.6 & 20.21 & 4.6 & -24.69 & -21.62 & -24.75 & 0.059 \\
\hline A0970 & 26.34 & 97.5 & 6.1 & 20.98 & 2.5 & -24.04 & -20.52 & -24.08 & 0.039 \\
\hline A 0978 & 24.11 & 51.3 & 3.1 & 19.74 & 2.6 & -24.50 & -21.79 & -24.58 & 0.083 \\
\hline A0979 & 24.11 & 36.7 & 5.0 & 20.74 & 2.2 & -24.01 & -20.50 & -24.05 & 0.039 \\
\hline A0999 & 23.77 & 28.4 & 6.1 & 19.73 & 1.9 & -23.71 & -20.90 & -23.79 & 0.075 \\
\hline A1003 & 22.78 & 20.3 & 1.5 & 19.45 & 1.7 & -23.53 & -21.26 & -23.66 & 0.123 \\
\hline A1016 & 23.26 & 17.5 & 5.9 & 19.57 & 1.5 & -23.16 & -20.65 & -23.26 & 0.099 \\
\hline A 1020 & 23.68 & 24.5 & 3.9 & 20.14 & 2.5 & -23.49 & -21.40 & -23.64 & 0.145 \\
\hline A1066 & 25.96 & 159.9 & 2.4 & 21.16 & 7.2 & -25.05 & -22.70 & -25.17 & 0.115 \\
\hline A1069 & 22.80 & 26.3 & 2.0 & 20.06 & 2.4 & -24.18 & -21.37 & -24.25 & 0.075 \\
\hline A 1100 & 25.24 & 74.8 & 6.3 & 20.61 & 3.0 & -24.52 & -21.22 & -24.57 & 0.048 \\
\hline A1139 & 24.75 & 52.6 & 5.3 & 19.76 & 1.7 & -24.07 & -20.79 & -24.12 & 0.049 \\
\hline A1142 & 24.61 & 52.3 & 5.1 & 19.82 & 2.0 & -24.12 & -20.96 & -24.18 & 0.055 \\
\hline A1149 & 24.61 & 49.6 & 5.0 & 18.58 & 1.3 & -24.25 & -21.61 & -24.34 & 0.088 \\
\hline A 1155 & 24.10 & 31.3 & 3.5 & 20.41 & 2.6 & -23.61 & -21.30 & -23.73 & 0.120 \\
\hline A 1171 & 25.40 & 70.1 & 3.6 & 21.09 & 4.3 & -24.05 & -21.66 & -24.16 & 0.111 \\
\hline A1185 & 23.56 & 29.8 & 4.2 & 21.01 & 2.6 & -23.86 & -20.43 & -23.91 & 0.042 \\
\hline A1187 & 22.87 & 22.1 & 1.9 & 20.08 & 3.0 & -23.79 & -21.93 & -23.97 & 0.180 \\
\hline A 1203 & 22.88 & 20.5 & 3.7 & 21.09 & 1.8 & -23.94 & -19.82 & -23.97 & 0.022 \\
\hline A 1216 & 22.89 & 15.7 & 3.5 & 19.78 & 1.8 & -23.21 & -21.00 & -23.34 & 0.130 \\
\hline A 1228 & 23.88 & 25.1 & 5.7 & 19.34 & 1.5 & -23.34 & -20.89 & -23.45 & 0.105 \\
\hline A 1238 & 25.50 & 92.3 & 4.0 & 20.11 & 2.3 & -24.59 & -21.24 & -24.63 & 0.046 \\
\hline A 1257 & 21.74 & 6.9 & 1.4 & 18.32 & 1.1 & -21.96 & -21.20 & -22.40 & 0.495 \\
\hline A1308 & 25.48 & 70.4 & 6.5 & 21.05 & 2.6 & -24.21 & -20.55 & -24.25 & 0.034 \\
\hline
\end{tabular}


TABle 3-Continued

\begin{tabular}{|c|c|c|c|c|c|c|c|c|c|}
\hline $\begin{array}{l}\text { Name } \\
(1)\end{array}$ & $\begin{array}{l}\mu_{e} \\
(2)\end{array}$ & $\begin{array}{l}r_{e} \\
(3)\end{array}$ & $\begin{array}{c}n \\
(4)\end{array}$ & $\begin{array}{l}\mu_{0} \\
(5)\end{array}$ & $\begin{array}{l}r_{0} \\
(6)\end{array}$ & $\begin{array}{c}M_{\text {Sersic }} \\
(7)\end{array}$ & $\begin{array}{c}M_{\text {exp }} \\
\text { (8) }\end{array}$ & $\begin{array}{l}M_{T} \\
(9)\end{array}$ & $\begin{array}{l}e / S \\
(10)\end{array}$ \\
\hline A 1314 & 24.79 & 77.1 & 4.0 & 19.18 & 2.2 & -24.66 & -21.86 & -24.73 & 0.076 \\
\hline A 1317 & 25.12 & 137.6 & 2.4 & 20.74 & 4.8 & -25.57 & -22.23 & -25.62 & 0.046 \\
\hline A 1424 & 21.93 & 14.6 & 1.7 & 19.19 & 1.9 & -23.77 & -21.87 & -23.95 & 0.172 \\
\hline $\mathrm{A} 1474$ & 21.45 & 12.3 & 1.0 & 19.65 & 1.6 & -23.68 & -21.09 & -23.78 & 0.092 \\
\hline A 1507 & 22.83 & 22.4 & 1.5 & 19.88 & 2.7 & -23.68 & -21.86 & -23.87 & 0.187 \\
\hline A1520 & 24.64 & 85.8 & 4.2 & 19.94 & 2.8 & -25.31 & -21.86 & -25.36 & 0.042 \\
\hline A 1534 & 24.13 & 41.4 & 5.5 & 20.55 & 3.1 & -24.41 & -21.50 & -24.48 & 0.069 \\
\hline A1569 & 21.95 & 17.1 & 1.5 & 19.36 & 2.0 & -24.04 & -21.76 & -24.17 & 0.122 \\
\hline A 1610 & 24.34 & 42.5 & 7.3 & 19.86 & 1.8 & -24.36 & -20.91 & -24.41 & 0.042 \\
\hline A1648 & 23.72 & 43.6 & 1.0 & 20.55 & 5.2 & -24.06 & -22.62 & -24.32 & 0.266 \\
\hline A1749 & 23.59 & 35.7 & 3.6 & 19.78 & 2.9 & -24.34 & -22.07 & -24.46 & 0.124 \\
\hline A1773 & 23.91 & 47.2 & 2.3 & 20.27 & 3.4 & -24.47 & -22.00 & -24.58 & 0.102 \\
\hline A 1775 & 23.22 & 31.5 & 1.5 & 20.76 & 5.3 & -24.04 & -22.42 & -24.26 & 0.224 \\
\hline A1795 & 27.15 & 354.7 & 6.2 & 20.98 & 3.5 & -26.09 & -21.33 & -26.11 & 0.012 \\
\hline A1809 & 24.65 & 76.6 & 3.8 & 20.12 & 3.5 & -25.03 & -22.16 & -25.11 & 0.071 \\
\hline A 1831 & 23.96 & 63.7 & 3.0 & 20.23 & 2.7 & -25.23 & -21.54 & -25.27 & 0.033 \\
\hline A1904 & 23.73 & 45.6 & 3.6 & 19.97 & 2.3 & -24.82 & -21.43 & -24.86 & 0.044 \\
\hline A1964 & 24.18 & 44.1 & 2.1 & 20.01 & 2.8 & -23.97 & -21.75 & -24.10 & 0.130 \\
\hline A1982 & 22.43 & 19.8 & 1.0 & 19.98 & 3.2 & -23.56 & -22.08 & -23.81 & 0.255 \\
\hline A2022 & 24.80 & 65.6 & 3.7 & 19.72 & 2.5 & -24.45 & -21.81 & -24.54 & 0.088 \\
\hline A2028 & 25.46 & 100.1 & 6.2 & 21.10 & 3.8 & -25.06 & -21.37 & -25.09 & 0.033 \\
\hline A 2040 & 27.66 & 561.3 & 5.0 & 19.87 & 2.2 & -26.34 & -21.29 & -26.35 & 0.010 \\
\hline A 2147 & 28.07 & 691.6 & 5.8 & 21.17 & 5.5 & -26.45 & -21.97 & -26.47 & 0.016 \\
\hline A 2151 & 22.94 & 25.4 & 2.1 & 19.55 & 1.9 & -23.77 & -21.20 & -23.87 & 0.093 \\
\hline A 2162 & 23.02 & 26.1 & 3.8 & 19.33 & 1.9 & -24.02 & -21.31 & -24.11 & 0.083 \\
\hline A 2197 & 22.23 & 23.8 & 2.6 & 19.21 & 2.6 & -24.37 & -22.11 & -24.50 & 0.125 \\
\hline A2199 & 24.12 & 58.2 & 2.9 & 21.38 & 5.6 & -24.50 & -21.64 & -24.57 & 0.072 \\
\hline A2309 & 23.92 & 31.3 & 4.0 & 19.46 & 1.9 & -23.72 & -21.39 & -23.84 & 0.117 \\
\hline A2319 & 22.72 & 31.0 & 1.7 & 20.71 & 1.9 & -24.58 & -20.22 & -24.60 & 0.018 \\
\hline A2331 & 24.38 & 51.1 & 3.2 & 20.32 & 1.8 & -24.35 & -20.52 & -24.38 & 0.029 \\
\hline A 2366 & 23.62 & 35.0 & 3.0 & 19.58 & 2.8 & -24.14 & -22.15 & -24.30 & 0.160 \\
\hline A 2372 & 25.34 & 64.3 & 7.2 & 20.68 & 3.7 & -24.22 & -21.67 & -24.32 & 0.095 \\
\hline A 2457 & 24.65 & 71.8 & 4.8 & 20.76 & 3.8 & -24.93 & -21.66 & -24.98 & 0.049 \\
\hline A2462 & 25.41 & 86.2 & 4.6 & 19.83 & 2.6 & -24.71 & -21.90 & -24.79 & 0.075 \\
\hline A2480 & 26.55 & 144.4 & 7.5 & 19.60 & 2.2 & -24.92 & -21.73 & -24.98 & 0.053 \\
\hline A 2492 & 23.84 & 30.6 & 4.1 & 20.70 & 2.8 & -23.95 & -21.19 & -24.03 & 0.079 \\
\hline A 2524 & 23.20 & 25.6 & 2.3 & 20.34 & 2.9 & -23.95 & -21.68 & -24.07 & 0.124 \\
\hline A 2559 & 23.14 & 41.4 & 2.2 & 20.31 & 2.5 & -24.92 & -21.29 & -24.96 & 0.035 \\
\hline A 2572 & 28.80 & 602.5 & 9.1 & 19.36 & 2.2 & -25.67 & -21.82 & -25.70 & 0.029 \\
\hline A 2572 & 26.02 & 131.5 & 5.2 & 19.25 & 2.3 & -24.84 & -21.95 & -24.91 & 0.070 \\
\hline A 2593 & 22.92 & 19.5 & 1.4 & 20.52 & 1.7 & -23.22 & -20.17 & -23.28 & 0.060 \\
\hline A 2626 & 22.92 & 29.0 & 2.3 & 19.18 & 0.8 & -24.40 & -19.93 & -24.41 & 0.016 \\
\hline A2634 & 26.93 & 283.2 & 7.8 & 19.56 & 1.7 & -25.77 & -21.01 & -25.78 & 0.012 \\
\hline A2656 & 23.03 & 22.8 & 2.0 & 19.69 & 1.7 & -23.77 & -21.19 & -23.87 & 0.093 \\
\hline A 2657 & 21.19 & 5.5 & 1.4 & 17.45 & 0.5 & -22.11 & -20.30 & -22.29 & 0.190 \\
\hline A2660 & 25.01 & 64.9 & 6.5 & 20.11 & 1.7 & -24.49 & -20.51 & -24.52 & 0.026 \\
\hline A 2670 & 23.54 & 40.8 & 2.6 & 19.84 & 2.8 & -24.57 & -21.99 & -24.67 & 0.092 \\
\hline A 2717 & 23.39 & 36.5 & 1.8 & 19.73 & 2.0 & -24.16 & -21.29 & -24.24 & 0.071 \\
\hline A 2734 & 25.48 & 127.8 & 3.9 & 22.45 & 8.1 & -25.27 & -21.62 & -25.30 & 0.035 \\
\hline A 2806 & 21.68 & 9.0 & 1.4 & 19.09 & 1.8 & -22.64 & -21.51 & -22.96 & 0.355 \\
\hline A 2841 & 23.70 & 35.1 & 4.1 & 19.77 & 2.2 & -24.28 & -21.48 & -24.36 & 0.076 \\
\hline A2859 & 23.75 & 25.0 & 3.6 & 20.30 & 2.4 & -23.42 & -21.13 & -23.54 & 0.122 \\
\hline A 2864 & 23.50 & 18.1 & 3.2 & 19.73 & 1.6 & -22.93 & -20.92 & -23.08 & 0.158 \\
\hline A 2877 & 22.94 & 37.7 & 4.1 & 18.62 & 1.8 & -24.78 & -21.80 & -24.85 & 0.064 \\
\hline A2881 & 23.03 & 12.8 & 3.7 & 19.62 & 1.6 & -22.70 & -20.92 & -22.89 & 0.195 \\
\hline A 2896 & 21.99 & 11.1 & 3.5 & 18.84 & 1.4 & -23.15 & -21.16 & -23.31 & 0.159 \\
\hline A3045 & 23.17 & 17.9 & 1.0 & 20.47 & 3.7 & -22.68 & -21.95 & -23.13 & 0.508 \\
\hline A3095 & 22.61 & 15.0 & 1.3 & 19.73 & 2.6 & -22.94 & -21.91 & -23.30 & 0.389 \\
\hline
\end{tabular}


TABLE 3-Continued

\begin{tabular}{|c|c|c|c|c|c|c|c|c|c|}
\hline $\begin{array}{c}\text { Name } \\
(1)\end{array}$ & $\begin{array}{l}\mu_{e} \\
(2)\end{array}$ & $\begin{array}{l}r_{e} \\
(3)\end{array}$ & $\begin{array}{c}n \\
(4)\end{array}$ & $\begin{array}{l}\mu_{0} \\
(5)\end{array}$ & $\begin{array}{l}r_{0} \\
(6)\end{array}$ & $\begin{array}{c}M_{\text {Sersic }} \\
(7)\end{array}$ & $\begin{array}{c}M_{e x p} \\
(8)\end{array}$ & $\begin{array}{r}M_{T} \\
(9)\end{array}$ & $\begin{array}{l}e / S \\
(10)\end{array}$ \\
\hline A3104 & 23.26 & 36.3 & 1.3 & 19.82 & 2.7 & -24.25 & -21.94 & -24.37 & 0.119 \\
\hline A3107 & 25.84 & 75.1 & 4.5 & 20.61 & 2.9 & -23.85 & -21.24 & -23.94 & 0.091 \\
\hline A3110 & 22.02 & 18.5 & 0.9 & 19.93 & 5.0 & -23.84 & -23.13 & -24.30 & 0.518 \\
\hline A3111 & 23.57 & 33.6 & 2.9 & 20.66 & 2.7 & -24.18 & -21.07 & -24.24 & 0.057 \\
\hline A3112 & 23.91 & 74.2 & 2.5 & 21.08 & 4.2 & -25.48 & -21.62 & -25.51 & 0.028 \\
\hline A3120 & 24.82 & 74.4 & 3.9 & 20.58 & 2.8 & -24.64 & -21.07 & -24.68 & 0.037 \\
\hline A 3122 & 24.29 & 44.8 & 6.0 & 19.48 & 2.3 & -24.43 & -21.85 & -24.53 & 0.093 \\
\hline A3123 & 21.98 & 11.5 & 1.3 & 18.95 & 1.7 & -22.97 & -21.74 & -23.27 & 0.322 \\
\hline A3125 & 22.59 & 11.8 & 0.9 & 19.85 & 2.6 & -22.25 & -21.74 & -22.78 & 0.625 \\
\hline A3142 & 24.48 & 47.9 & 4.8 & 20.69 & 2.7 & -24.26 & -21.05 & -24.32 & 0.052 \\
\hline A 3223 & 23.05 & 23.3 & 2.8 & 20.35 & 3.5 & -23.83 & -21.86 & -23.99 & 0.164 \\
\hline A 3225 & 22.16 & 12.9 & 1.1 & 19.58 & 2.3 & -22.93 & -21.75 & -23.24 & 0.337 \\
\hline A3266 & 22.83 & 43.6 & 1.3 & 19.92 & 3.1 & -25.01 & -22.04 & -25.08 & 0.064 \\
\hline A3301 & 26.53 & 240.0 & 5.2 & 20.89 & 5.2 & -25.70 & -22.20 & -25.74 & 0.040 \\
\hline A3332 & 28.15 & 326.5 & 9.2 & 20.84 & 3.7 & -25.16 & -21.63 & -25.20 & 0.039 \\
\hline A3336 & 24.45 & 61.0 & 2.8 & 21.20 & 5.0 & -24.60 & -21.89 & -24.69 & 0.082 \\
\hline A3341 & 24.76 & 57.7 & 4.5 & 19.29 & 1.4 & -24.22 & -20.80 & -24.26 & 0.043 \\
\hline A3354 & 26.09 & 80.0 & 6.8 & 20.56 & 3.4 & -23.93 & -21.63 & -24.05 & 0.121 \\
\hline A3374 & 20.79 & 6.5 & 1.0 & 17.99 & 0.6 & -22.72 & -20.43 & -22.85 & 0.121 \\
\hline A3376 & 25.10 & 85.9 & 5.2 & 20.43 & 3.3 & -24.89 & -21.63 & -24.95 & 0.050 \\
\hline A3380 & 28.80 & 593.9 & 7.4 & 20.62 & 3.3 & -25.57 & -21.44 & -25.60 & 0.022 \\
\hline A3390 & 24.31 & 39.8 & 4.2 & 19.74 & 1.6 & -23.84 & -20.68 & -23.89 & 0.055 \\
\hline A3408 & 25.06 & 82.1 & 5.1 & 20.16 & 2.8 & -24.78 & -21.52 & -24.84 & 0.049 \\
\hline A3420 & 23.56 & 19.3 & 2.8 & 20.43 & 2.7 & -22.91 & -21.29 & -23.13 & 0.224 \\
\hline A3528 & 24.78 & 92.0 & 3.8 & 19.92 & 2.7 & -25.30 & -21.83 & -25.34 & 0.041 \\
\hline A3530 & 24.04 & 67.3 & 3.2 & 19.93 & 2.1 & -25.25 & -21.28 & -25.28 & 0.026 \\
\hline A3531 & 24.96 & 56.2 & 3.2 & 19.73 & 1.2 & -23.85 & -20.09 & -23.88 & 0.031 \\
\hline A3532 & 28.22 & 728.6 & 6.5 & 20.37 & 2.8 & -26.64 & -21.44 & -26.65 & 0.008 \\
\hline A3554 & 23.45 & 41.2 & 2.5 & 19.18 & 1.3 & -24.60 & -20.96 & -24.64 & 0.035 \\
\hline A3559 & 22.75 & 29.3 & 2.0 & 18.85 & 1.9 & -24.45 & -22.08 & -24.56 & 0.113 \\
\hline A3562 & 28.77 & 1160.1 & 6.9 & 21.30 & 5.1 & -27.08 & -21.77 & -27.09 & 0.007 \\
\hline A 3574 & 23.36 & 48.2 & 3.3 & 18.52 & 1.0 & -24.53 & -20.40 & -24.55 & 0.022 \\
\hline A3605 & 27.28 & 305.7 & 4.1 & 21.47 & 8.2 & -25.42 & -22.67 & -25.51 & 0.079 \\
\hline A3667 & 28.77 & 812.0 & 8.6 & 21.39 & 5.8 & -26.37 & -21.91 & -26.39 & 0.016 \\
\hline A3703 & 25.45 & 101.2 & 3.6 & 21.19 & 4.3 & -24.78 & -21.54 & -24.83 & 0.051 \\
\hline A3716 & 23.49 & 40.6 & 2.6 & 19.79 & 2.1 & -24.49 & -21.31 & -24.55 & 0.053 \\
\hline A3731 & 25.84 & 94.8 & 7.1 & 20.15 & 2.7 & -24.53 & -21.47 & -24.59 & 0.060 \\
\hline A 3733 & 23.48 & 24.2 & 1.9 & 20.17 & 2.3 & -23.18 & -21.08 & -23.33 & 0.144 \\
\hline A3741 & 25.47 & 86.1 & 3.4 & 21.18 & 3.9 & -24.40 & -21.35 & -24.47 & 0.060 \\
\hline A3782 & 26.04 & 130.0 & 6.8 & 20.76 & 2.7 & -25.02 & -20.88 & -25.04 & 0.022 \\
\hline A3796 & 24.66 & 54.6 & 3.6 & 20.77 & 3.1 & -24.25 & -21.24 & -24.32 & 0.062 \\
\hline A3806 & 25.01 & 81.3 & 4.9 & 21.63 & 6.7 & -24.93 & -22.09 & -25.01 & 0.073 \\
\hline A3825 & 22.15 & 6.8 & 0.7 & 19.41 & 1.5 & -21.23 & -20.85 & -21.81 & 0.699 \\
\hline A3844 & 26.38 & 66.8 & 3.7 & 20.79 & 3.0 & -22.96 & -21.15 & -23.15 & 0.188 \\
\hline A3851 & 22.90 & 21.1 & 1.9 & 20.12 & 3.1 & -23.54 & -21.83 & -23.75 & 0.206 \\
\hline A3879 & 23.17 & 18.3 & 3.1 & 19.24 & 1.7 & -23.26 & -21.49 & -23.45 & 0.196 \\
\hline A3897 & 24.94 & 67.7 & 3.8 & 19.84 & 2.6 & -24.55 & -21.88 & -24.64 & 0.085 \\
\hline A3912 & 23.93 & 30.6 & 1.4 & 21.46 & 7.6 & -23.20 & -22.49 & -23.65 & 0.518 \\
\hline A 4049 & 23.85 & 31.5 & 4.4 & 19.63 & 1.8 & -23.77 & -21.03 & -23.86 & 0.080 \\
\hline
\end{tabular}

Note.-Col. (1), Abell Cluster; col (2), effective surface magnitude; col. (3), effective radius $(\mathrm{kpc})$; col. (4), $n$ parameter; col. (5), central surface magnitude; col. (6), scale length (kpc); col. (7), Sersic absolute magnitude; col. (8), exponential absolute magnitude; col. (9), total absolute magnitude; col. (10), exponential/Sérsic ratio. 